\title{
NUMERICAL ESTIMATION OF A DIFFUSION COEFFICIENT IN SUBDIFFUSION*
}

\author{
BANGTI $\mathrm{JIN}^{\dagger}$ AND ZHI ZHOU ${ }^{\ddagger}$
}

\begin{abstract}
In this work, we consider the numerical recovery of a spatially dependent diffusion coefficient in a subdiffusion model from distributed observations. The subdiffusion model involves a Caputo fractional derivative of order $\alpha \in(0,1)$ in time. The numerical estimation is based on the regularized output least-squares formulation, with an $H^{1}(\Omega)$ penalty. We prove the well-posedness of the continuous formulation, e.g., existence and stability. Next, we develop a fully discrete scheme based on the Galerkin finite element method in space and backward Euler convolution quadrature in time. We prove the subsequential convergence of the sequence of discrete solutions to a solution of the continuous problem as the discretization parameters (mesh size and time step size) tend to zero. Further, under an additional regularity condition on the exact coefficient, we derive convergence rates in a weighted $L^{2}(\Omega)$ norm for the discrete approximations to the exact coefficient in the one- and two-dimensional cases. The analysis relies heavily on suitable nonstandard nonsmooth data error estimates for the direct problem. We provide illustrative numerical results to support the theoretical study.
\end{abstract}

Key words. parameter identification, subdiffusion, fully discrete scheme, convergence, error estimate, Tikhonov regularization

AMS subject classifications. 35R11, 35R30, 49M25, 65M60

DOI. $10.1137 / 19 \mathrm{M} 1295088$

1. Introduction. Let $\Omega \subset \mathbb{R}^{d}(d=1,2,3)$ be a convex polyhedral domain with a boundary $\partial \Omega$. Consider the following initial-boundary value problem of the subdiffusion equation:

$$
\left\{\begin{aligned}
\partial_{t}^{\alpha} u(x, t)-\nabla \cdot(q(x) \nabla u(x, t)) & =f(x, t), & & (x, t) \in \Omega \times(0, T], \\
u(x, 0) & =u_{0}(x), & & x \in \Omega, \\
u(x, t) & =0, & & (x, t) \in \partial \Omega \times(0, T],
\end{aligned}\right.
$$

where $T>0$ is the final time. The functions $f$ and $u_{0}$ are the given source term and initial condition, respectively, and their precise regularity will be specified below. The notation $\partial_{t}^{\alpha} u$ denotes the Caputo fractional derivative in time of order $\alpha \in(0,1)$, defined by [33]

$$
\partial_{t}^{\alpha} u(t)=\frac{1}{\Gamma(1-\alpha)} \int_{0}^{t}(t-s)^{-\alpha} u^{\prime}(s) \mathrm{d} s .
$$

The fractional derivative $\partial_{t}^{\alpha} u$ recovers the usual first-order derivative $u^{\prime}(s)$ as the order $\alpha \rightarrow 1^{-}$for smooth functions $u$. Thus the model is a fractional analogue of the classical diffusion model. Throughout, we denote the solution to problem (1.1) by $u(q)$ to explicitly indicate its dependence on the diffusion coefficient $q$.

\footnotetext{
* Received by the editors October 23, 2019; accepted for publication (in revised form) January 8, 2021; published electronically April 15, 2021.

https://doi.org/10.1137/19M1295088

Funding: The work of the first author is supported by UK EPSRC grant EP/T000864/1. The research of the second author is supported by Hong Kong RGC grant 25300818.

${ }^{\dagger}$ Department of Computer Science, University College London, London, WC1E 6BT, UK (bangti.jin@gmail.com).

${ }^{\ddagger}$ Department of Applied Mathematics, The Hong Kong Polytechnic University, Kowloon, Hong Kong (zhi.zhou@polyu.edu.hk).
} 
The model (1.1) has received enormous attention in recent years, due to its extraordinary capability for describing anomalously slow diffusion processes (also known as subdiffusion), which displays local motion occasionally interrupted by long sojourns and trapping effects. At a microscopic level, such anomalous diffusion processes are accurately modeled by continuous time random walk, where the waiting time between consecutive particle jumps follows a heavy tailed distribution with a divergent mean, and the model (1.1) is the macroscopic counterpart, describing the evolution of the probability density function (in $\mathbb{R}^{d}$ ) of the particle appearing at time $t$ and spatical location $t$, in analogy to Brownian motion for normal diffusion. These processes are characterized by sublinear growth of the particle mean squared displace with the time. The model (1.1) has found many applications in physics, biology, finance, etc., including electron transport with copier [48], thermal diffusion on fractal domains [43], dispersive transport of ions in column experiments [1, 18], protein transport within membranes [35, 34, 46], and solute transport in heterogeneous media [11, 5]. We refer interested readers to the comprehensive reviews $[6,42,41]$ for physical modeling and long lists of diverse applications.

This work is concerned with numerically identifying the diffusion coefficient $q^{\dagger} \in$ $L^{\infty}(\Omega)$ the model (1.1) from the (noisy) distributed observation

$$
z^{\delta}(x, t)=u\left(q^{\dagger}\right)(x, t)+\xi(x, t), \quad(x, t) \in \Omega \times[0, T],
$$

where $u\left(q^{\dagger}\right)$ is the exact data (corresponding to the exact diffusion coefficient $q^{\dagger}$ ), and $\xi$ denotes the noise, with an accuracy $\delta=\left\|u\left(q^{\dagger}\right)-z^{\delta}\right\|_{L^{2}\left(0, T ; L^{2}(\Omega)\right)}$. The inverse problem is a fractional analogue of the inverse conductivity problem for standard parabolic problems, which has been extensively studied both numerically and theoretically; see the monograph [19, Chapter 9] for relevant mathematical theory and the references $[17,29,31,14,12,44,50]$ for a rather incomplete list of works on numerical identification of a diffusion coefficient in standard parabolic problems. Most of these existing works formulate the inverse problem into an output least-squares formulation, with a suitable penalty, e.g., Sobolev smoothness or total variation. Formally, the inverse problem is overdetermined for uniqueness/identifiability, and the terminal data at time $T$ or lateral Cauchy data may suffice for unique recovery (see [19, Chapter 9] for relevant uniqueness results for a standard parabolic case). Nonetheless, numerically, the full space-time datum (1.2) or a restricted version over the region $\Omega \times\left[T_{0}, T\right]$ is frequently employed in existing studies for standard parabolic problems $[17,49,29,31,14,12,44,50]$, due to, e.g., the weak regularity assumption on problem data. In particular, all existing works [31, 44, 50] on error estimates (for parabolic problems) require the full space-time datum (1.2), and it appears to be open to extend these results to partial data. Thus, we shall focus the analysis on the full datum (1.2) below.

In this work, we shall develop a numerical procedure for recovering a spatially dependent diffusion coefficient. We formulate an output least-squares formulation with an $H^{1}(\Omega)$ penalty, which is suitable for recovering a smooth coefficient $q$, and provide a complete analysis of both continuous and discrete formulations, including well-posedness and convergence of discrete approximations, for weak regularity assumption on the problem data, in sections 2 and 3, respectively. Furthermore, in section 4 , we derive some a priori weighted $L^{2}(\Omega)$ error estimates on the discrete approximation under a mild regularity assumption on the exact diffusion coefficient $q^{\dagger}$ in one- and two-dimensional cases; see Theorem 4.8 and Corollary 4.10. The obtained estimates depend on the spatial mesh size $h$, temporal step size $\tau$, the noise level $\delta$, and the regularization parameter $\gamma$. These results extend the corresponding 
results for the standard parabolic case $[17,31,50]$ and represent the main theoretical achievements of the work.

Generally, when compared with standard parabolic problems, the presence of the time-fractional derivative $\partial_{t}^{\alpha} u$ in the model (1.1) poses a number of distinct challenges to the mathematical and numerical analysis (see [23] for a concise overview): (i) due to the nonlocality of the Caputo derivative $\partial_{t}^{\alpha} u$, many powerful tools from PDE theory and classical numerical analysis, e.g., energy argument and integration by parts formula, are not directly applicable; (ii) the solution $u$ generally has only limited spatial and temporal regularity, even for smooth problem data; (iii) high-order time stepping schemes often lack robustness with respect to the regularity of the problem data; (iv) the nonlocality incurs a storage issue for time stepping. Naturally, these challenges persist for the analysis of the regularized output least-squares formulation (2.1)-(2.2) below, and especially items (i) and (ii) represent the main technical challenges in the convergence analysis, and hence it differs substantially from the standard parabolic counterpart. Further, the error analysis is greatly complicated by the nonlinearity of the forward map, and thus standard techniques from optimal control theory, via, e.g., convexity and the first-order optimality condition, also do not apply directly. To overcome these technical challenges, we shall employ the positivity of the fractional derivative operators (in Lemmas 2.2 and 3.1), nonsmooth data estimates (in Lemma 4.2), and novel test function $\varphi$ (in Theorem 4.8), which represent the main technical novelties of the work.

Now we briefly review relevant works from the inverse problem literature. Inverse problems for fractional diffusion have started to attract much interest, and there has already been a vast literature (see, e.g., the review [26]). There are a number of interesting works on recovering the diffusion coefficient $[8,36,37,52,32]$. In an influential work, Cheng et al. [8] proved the unique recovery of both diffusion coefficient and fractional order from the lateral Cauchy data for the model (1.1) with a Dirac source in the one spatial dimensional case. The proof employs Laplace transform and Sturm-Liouville theory. Recently, Kian et al. [32] proved uniqueness for the recovery of two coefficients from the Dirichlet-to-Neumann map [32]. Li and co-authors [36, 37] discussed the numerical recovery of the diffusion coefficient (simultaneously with the fractional order) and showed various continuity results of the parameter to state map. However, the numerical discretization was not analyzed in [37]. Zhang [52] proved the unique recovery for the case of a time-dependent $q \equiv q(t)$ and devised a numerical scheme for its recovery. See also the work [51] for further numerical results on recovering the diffusion coefficient from boundary data in the one-dimensional case, using a space-time variational formulation, which allows only a zero initial condition. However, there is neither analysis of the discretized problem nor error estimates in these interesting existing works. In sum, there is no rigorous study of the discretization schemes, and it is precisely this gap that this work aims to fill in. We refer interested readers also to the works [53, 21, 28] and references therein for further numerical methods on other nonlinear inverse problems for the subdiffusion model.

The rest of the paper is organized as follows. In section 2 , we formulate the continuous problem and analyze its well-posedness, e.g., existence and stability. Then in section 3, we describe a fully discrete scheme and show the convergence of the discrete approximations to a solution of the continuous problem as the discretization parameters tend to zero. In section 4 , we provide detailed error estimates for the discrete approximations. Finally, in section 5, we present illustrative one- and twodimensional numerical results to complement the analysis. We conclude the paper with further remarks in section 6 . 
We end this section with some useful notation. Throughout, the notation $c$ denotes a generic constant, which may change at each occurrence, but it is always independent of $q$, mesh size $h$, and time step size $\tau$. We shall employ standard notation for Sobolev spaces [2]. The spaces $L^{p}(\Omega)$ and $W^{k, p}(\Omega)$ are endowed with the norms $\|\cdot\|_{L^{p}(\Omega)}$ and $\|\cdot\|_{W^{k, p}(\Omega)}$, respectively, and the notation $(\cdot, \cdot)$ denotes the $L^{2}(\Omega)$ inner product. We denote by $H^{-1}(\Omega)$ the dual space of $H_{0}^{1}(\Omega)$. For a Banach space $B$ (endowed with the norm $\|\cdot\|_{B}$ ), we define $L^{2}(0, T ; B)=\{u(t) \in$ $B$ for a.e. $t \in(0, T)$ and $\left.\|u\|_{L^{2}(0, T ; B)}<\infty\right\}$, and the norm is given by $\|u\|_{L^{2}(0, T ; B)}=$ $\left(\int_{0}^{T}\|u(t)\|_{B}^{2} \mathrm{~d} t\right)^{\frac{1}{2}}$. The notation $\left.(\cdot, \cdot)_{L^{2}\left(0, T ; L^{2}(\Omega)\right.}\right)$ denotes the inner product in the space $L^{2}\left(0, T ; L^{2}(\Omega)\right)$. Similarly, the space $H^{1}(0, T ; B)$ denotes $H^{1}(0, T ; B)=\{u \in$ $\left.L^{2}(0, T ; B): u^{\prime}(t) \in L^{2}(0, T ; B)\right\}$, with its norm given by $\|u\|_{H^{1}(0, T ; B)}=\left(\|u\|_{L^{2}(0, T ; B)}^{2}+\right.$ $\left.\left\|u^{\prime}(t)\right\|_{L^{2}(0, T ; B)}^{2}\right)^{\frac{1}{2}}$, with the notation ' denoting the (weak) temporal derivative. Further, for any $s \geq 0$, we denote by $\dot{H}^{s}(\Omega)=\left\{v \in L^{2}(\Omega):(-\Delta)^{\frac{s}{2}} v \in L^{2}(\Omega)\right\}$, where $\Delta$ is the Laplacian with a zero Dirichlet boundary condition and the fractional power $(-\Delta)^{\frac{s}{2}}$ is defined by the spectral decomposition [30]. The space $\dot{H}^{s}(\Omega)$ is equipped with the norm $\|v\|_{\dot{H}^{s}(\Omega)}=\left(\|v\|_{L^{2}(\Omega)}^{2}+\left\|(-\Delta)^{\frac{s}{2}} v\right\|_{L^{2}(\Omega)}^{2}\right)^{\frac{1}{2}}$. Then $\dot{H}^{0}(\Omega)=L^{2}(\Omega)$, $\dot{H}^{1}(\Omega)=H_{0}^{1}(\Omega)$ and $\dot{H}^{2}(\Omega)=H^{2}(\Omega) \cap H_{0}^{1}(\Omega)$.

2. Well-posedness of the continuous problem. In this section, we formulate and analyze the continuous formulation of the reconstruction approach. To recover the diffusion coefficient $q$, we employ the following output least-squares formulation with an $H^{1}(\Omega)$-penalty:

$$
\min _{q \in \mathcal{A}} J_{\gamma}\left(q ; z^{\delta}\right)=\frac{1}{2}\left\|u(q)-z^{\delta}\right\|_{L^{2}\left(0, T ; L^{2}(\Omega)\right)}^{2}+\frac{\gamma}{2}\|\nabla q\|_{L^{2}(\Omega)}^{2},
$$

where $u(q)$ satisfies the variational problem

$$
\left(\partial_{t}^{\alpha} u(q), v\right)+(q \nabla u(q), \nabla v)=(f, v) \quad \forall v \in \dot{H}^{1}(\Omega), t \in(0, T] \quad \text { with } \quad u(0)=u_{0} .
$$

The admissible set $\mathcal{A}$ for the diffusion coefficient $q(x)$ is given by

$$
\mathcal{A}=\left\{q \in H^{1}(\Omega): \quad c_{0} \leq q \leq c_{1} \text { a.e. in } \Omega\right\}
$$

with constants $c_{0}, c_{1} \in \mathbb{R}$ and $0<c_{0}<c_{1}$. The $H^{1}(\Omega)$ seminorm penalty is suitable for recovering a smooth diffusion coefficient. In the case of nonsmooth coefficients, alternative penalties, e.g., total variation, should be employed; see Remark 3.8 for further discussions. The scalar $\gamma>0$ is the regularization parameter, controlling the strength of the penalty [20]. The dependence of the functional $J_{\gamma}$ on $z^{\delta}$ will be suppressed below whenever there is no confusion. For the analysis in sections 2 and 3 , we make the following assumption on problem data. It is sufficient to ensure the existence of a unique solution $u(q) \in L^{2}\left(0, T ; H^{1}(\Omega)\right)$ for any $q \in \mathcal{A}$ [23].

Assumption 2.1. $u_{0} \in \dot{H}^{1}(\Omega), f \in L^{2}\left(0, T ; L^{2}(\Omega)\right)$, and $z^{\delta} \in L^{2}\left(0, T ; L^{2}(\Omega)\right)$.

First we show the well-posedness of problem $(2.1)-(2.2)$, which relies on a continuity result for the parameter-to-state map $u(q)$. First, we recall a stability result on the solution operator. Below, for any $q \in \mathcal{A}$, the operator $A(q): \dot{H}^{1}(\Omega) \rightarrow H^{-1}(\Omega)$ is defined by

$$
-\langle A(q) \varphi, \psi\rangle=(q \nabla \varphi, \nabla \psi) \quad \forall \varphi, \psi \in \dot{H}^{1}(\Omega),
$$

where $\langle\cdot, \cdot\rangle$ denotes the duality pairing between $H^{-1}(\Omega)$ and $\dot{H}^{1}(\Omega)$. For any $\varphi \in$ $\dot{H}^{2}(\Omega)$, there holds $A(q) \varphi=\nabla \cdot(q \nabla \varphi) \in L^{2}(\Omega)$. 
Lemma 2.2. For any $q \in \mathcal{A}$, let $v$ solve

$$
\partial_{t}^{\alpha} v-A(q) v=f \quad \forall t \in(0, T] \quad \text { with } v(0)=0 .
$$

Then there holds

$$
\|v\|_{L^{2}\left(0, T ; H^{1}(\Omega)\right)}^{2} \leq c\|f\|_{L^{2}\left(0, T ; H^{-1}(\Omega)\right)}^{2} .
$$

Proof. Taking $\phi=v$ in the weak formulation and then integrating from 0 to $T$ gives

$$
\left(\partial_{t}^{\alpha} v(t), v(t)\right)_{L^{2}\left(0, T ; L^{2}(\Omega)\right)}+(q \nabla v, \nabla v)_{L^{2}\left(0, T ; L^{2}(\Omega)\right)}=(f, v)_{L^{2}\left(0, T ; L^{2}(\Omega)\right)} .
$$

Since $v(0)=0$, the Caputo fractional derivative coincides with the Riemann-Liouville one [33], and upon extending $v$ by 0 outside [0,T], it follows directly from [38, Lemma 2.3] that

$$
\left(\partial_{t}^{\alpha} v(t), v(t)\right)_{L^{2}\left(0, T ; L^{2}(\Omega)\right)} \geq 0
$$

and by Poincaré's inequality and the Cauchy-Schwarz inequality, we obtain the desired estimate.

The next result gives the continuity of the parameter-to-state map.

Lemma 2.3. If the sequence $\left\{q^{n}\right\} \subset \mathcal{A}$ converges to $q \in \mathcal{A}$ almost everywhere, then

$$
\lim _{n \rightarrow \infty}\left\|u(q)-u\left(q^{n}\right)\right\|_{L^{2}\left(0, T ; H^{1}(\Omega)\right)}=0 .
$$

Proof. Let $v^{n}=u(q)-u\left(q^{n}\right)$. Then it satisfies $v^{n}(0)=0$ and

$$
\partial_{t}^{\alpha} v^{n}-\nabla \cdot\left(q^{n} \nabla v^{n}\right)=\nabla \cdot\left(\left(q-q^{n}\right) \nabla u(q)\right) \quad \forall t \in(0, T] .
$$

Then by Lemma 2.2 and the definition of the $H^{-1}(\Omega)$, we obtain

$$
\begin{aligned}
\left\|v^{n}\right\|_{L^{2}\left(0, T ; H^{1}(\Omega)\right)} & \leq c\left\|\nabla \cdot\left(\left(q-q^{n}\right) \nabla u(q)\right)\right\|_{L^{2}\left(0, T ; H^{-1}(\Omega)\right)} \\
& \leq c\left\|\left(q-q^{n}\right) \nabla u(q)\right\|_{L^{2}\left(0, T ; L^{2}(\Omega)\right)} .
\end{aligned}
$$

Let $\phi^{n}=\left|q-q^{n}\right|^{2} \int_{0}^{T}|\nabla u(q)|^{2} \mathrm{~d} t$. Then $\phi^{n} \rightarrow 0$ almost everywhere (a.e.), since $q^{n} \rightarrow q$ a.e., and further, since $q, q^{n} \in \mathcal{A}$, we have $0 \leq \phi^{n} \leq 4 c_{1}^{2} \int_{0}^{T}|\nabla u(q)|^{2} \mathrm{~d} t \in L^{1}(\Omega)$. Then, Lebesgue's dominated convergence theorem [16, Theorem 1.9] implies

$$
\lim _{n \rightarrow \infty}\left\|\left(q-q^{n}\right) \nabla u(q)\right\|_{L^{2}\left(0, T ; L^{2}(\Omega)\right)}^{2}=\lim _{n \rightarrow \infty} \int_{\Omega} \phi^{n}(x) \mathrm{d} x=\int_{\Omega} \lim _{n \rightarrow \infty} \phi^{n}(x) \mathrm{d} x=0,
$$

which shows the desired estimate.

The next result gives the existence of a minimizer. With Lemma 2.3 at hand, the result follows by a standard compactness argument in calculus of variation (see, e.g., $[13,20])$, and the proof is included only for completeness.

TheOREm 2.4. Under Assumption 2.1, there exists at least one minimizer to $(2.1)-(2.2)$.

Copyright $@$ ㅇ by SIAM. Unauthorized reproduction of this article is prohibited. 
Proof. Since the functional $J_{\gamma}$ is bounded from below by zero, there exists a minimizing sequence $\left\{q^{n}\right\}_{n \geq 1} \subset \mathcal{A}$ such that $\lim _{n \rightarrow \infty} J_{\gamma}\left(q^{n}\right)=\inf _{q \in \mathcal{A}} J_{\gamma}(q)$. Thus, the sequence $\left\{q^{n}\right\}_{n \geq 1}$ is uniformly bounded in the $H^{1}(\Omega)$ seminorm, which together with the box constraint $q^{n} \in \mathcal{A}$ implies that it is also uniformly bounded in $H^{1}(\Omega)$. Thus there exists a subsequence, still denoted by $\left\{q^{n}\right\}_{n>1}$, that converges to some $q^{*} \in$ $\mathcal{A}$ weakly in $H^{1}(\Omega)$, and by compact Sobolev embedding theorem [16], converges also in $L^{1}(\Omega)$. Further, by standard measure theory, convergence in $L^{1}(\Omega)$ implies almost everywhere convergence up to a subsequence [16, Theorem 1.21, p. 29]. Thus, we may assume that the subsequence $\left\{q^{n}\right\}_{n \geq 1}$ converges to $q^{*}$ in $L^{1}(\Omega)$ and almost everywhere. Then by Lemma 2.3, for the sequence $\left\{u\left(q^{n}\right)\right\}_{n \geq 1}$ of solutions to problem (1.1), there holds $\lim _{n \rightarrow \infty}\left\|u\left(q^{n}\right)-u\left(q^{*}\right)\right\|_{L^{2}\left(0, T ; H^{1}(\Omega)\right)}=0$. Then by Sobolev embedding [2], $\lim _{n \rightarrow \infty}\left\|u\left(q^{n}\right)-z^{\delta}\right\|_{L^{2}\left(0, T ; L^{2}(\Omega)\right)}^{2}=\left\|u\left(q^{*}\right)-z^{\delta}\right\|_{L^{2}\left(0, T ; L^{2}(\Omega)\right)}^{2}$. This and weak lower semicontinuity of seminorms imply that $q^{*}$ is a minimizer to (2.1).

The following continuous dependence results hold, where the minimum $H^{1}(\Omega)$ seminorm solution refers to the solution $q^{\dagger}$ of the minimum $H^{1}(\Omega)$ seminorm among all solutions within the admissible set $\mathcal{A}$ corresponding to the exact data $z^{\dagger}=u\left(q^{\dagger}\right)$. The proof follows by a standard argument (see, e.g., $[13,20]$ ) and thus is omitted.

TheOREM 2.5. Under Assumption 2.1, the following statements hold.

(i) Let $\gamma>0$ be fixed, the sequence $\left\{z_{j}\right\}_{j \geq 1}$ be convergent to some data $z$ in $L^{2}\left(0, T ; L^{2}(\Omega)\right)$, and $q_{j}^{*} \in \mathcal{A}$ the corresponding minimizer to $J_{\gamma}\left(\cdot ; z_{j}\right)$. Then $\left\{q_{j}^{*}\right\}_{j \geq 1}$ contains a subsequence convergent to a minimizer of $J_{\gamma}(\cdot ; z)$ over $\mathcal{A}$ in $H^{1}(\Omega)$.

(ii) Let $\left\{\delta_{j}\right\}_{j \geq 1} \subset \mathbb{R}_{+}$with $\delta_{j} \rightarrow 0^{+},\left\{z^{\delta_{j}}\right\}_{j \geq 1} \subset L^{2}\left(0, T ; L^{2}(\Omega)\right)$ be a sequence satisfying $\left\|z^{\delta_{j}}-z^{\dagger}\right\|_{L^{2}\left(0, T ; L^{2}(\Omega)\right)}=\delta_{j}$ for some exact data $z^{\dagger}=$ $u\left(q^{\dagger}\right)$, and $q_{j}^{*}$ be a minimizer to $J_{\gamma_{j}}\left(\cdot ; z^{\delta_{j}}\right)$ over $\mathcal{A}$. If $\left\{\gamma_{j}\right\}_{j \geq 1}$ satisfies

$\lim _{j \rightarrow \infty} \gamma_{j}=\lim _{j \rightarrow \infty} \frac{\delta_{j}^{2}}{\gamma_{j}}=0$, then $\left\{q_{j}^{*}\right\}_{j \geq 1}$ contains a subsequence converging to a minimum- $H^{1}(\Omega)$ seminorm solution in $H^{1}(\Omega)$.

3. Numerical approximation and convergence analysis. Now we describe the discretization of problem (2.1)-(2.2) and show the convergence of the approximations.

3.1. Numerical approximation. First, we describe a spatially semidiscrete scheme for problem (1.1) based on the Galerkin FEM; see [23] for a recent overview on the numerical approximation of the subdiffusion model. Let $\mathcal{T}_{h}$ be a shape regular quasi-uniform triangulation of the domain $\Omega$ into $d$-simplexes, denoted by $T$, with a mesh size $h \in(0,1)$. Over $\mathcal{T}_{h}$, we define a continuous piecewise linear finite element space $X_{h}$ by

$$
X_{h}=\left\{v_{h} \in \dot{H}^{1}(\Omega):\left.v_{h}\right|_{T} \text { is a linear function } \forall T \in \mathcal{T}_{h}\right\}
$$

and similarly the space $V_{h}$ by

$$
V_{h}=\left\{v_{h} \in H^{1}(\Omega):\left.v_{h}\right|_{T} \text { is a linear function } \forall T \in \mathcal{T}_{h}\right\} .
$$

The spaces $X_{h}$ and $V_{h}$ will be employed to approximate the state $u$ and the diffusion coefficient $q$, respectively. We define the $L^{2}(\Omega)$ projection $P_{h}: L^{2}(\Omega) \rightarrow X_{h}$ by

$$
\left(P_{h} \varphi, \chi\right)=(\varphi, \chi) \quad \forall \chi \in X_{h}
$$


Note that the operator $P_{h}$ satisfies the following error estimate: for any $s \in[1,2]$,

$$
\left\|P_{h} \varphi-\varphi\right\|_{L^{2}(\Omega)}+h\left\|\nabla\left(P_{h} \varphi-\varphi\right)\right\|_{L^{2}(\Omega)} \leq c h^{s}\|\varphi\|_{\dot{H}^{s}(\Omega)} \quad \forall \varphi \in \dot{H}^{s}(\Omega) .
$$

Let $\mathcal{I}_{h}$ be the interpolation operator associated with the finite element space $V_{h}$. Then it satisfies the following error estimates for $s=1,2$ (see, e.g., [15, Theorem 1.103]):

$$
\begin{aligned}
\left\|v-\mathcal{I}_{h} v\right\|_{L^{2}(\Omega)}+h\left\|v-\mathcal{I}_{h} v\right\|_{H^{1}(\Omega)} & \leq c h^{2}\|v\|_{H^{2}(\Omega)} \quad \forall v \in H^{2}(\Omega), \\
\left\|v-\mathcal{I}_{h} v\right\|_{L^{\infty}(\Omega)}+h\left\|v-\mathcal{I}_{h} v\right\|_{W^{1, \infty}(\Omega)} & \leq c h^{s}\|v\|_{W^{s, \infty}(\Omega)} \quad \forall v \in W^{s, \infty}(\Omega) .
\end{aligned}
$$

Now we partition the time interval $[0, T]$ uniformly, with grid points $t_{n}=n \tau$, $n=0, \ldots, N$, and a time step size $\tau=T / N$. The fully discrete scheme for problem (1.1) reads as follows: Given $U_{h}^{0}=P_{h} u_{0} \in X_{h}$, find $U_{h}^{n} \in X_{h}$ such that

$$
\left(\bar{\partial}_{\tau}^{\alpha}\left(U_{h}^{n}-U_{h}^{0}\right), \chi\right)+\left(q \nabla U_{h}^{n}, \nabla \chi\right)=\left(f^{n}, \chi\right) \quad \forall \chi \in X_{h}, \quad n=1,2, \ldots, N,
$$

where $f^{n}=\frac{1}{\tau} \int_{t_{n-1}}^{t_{n}} f(s) \mathrm{d} s$ and $\bar{\partial}_{\tau}^{\alpha} \varphi^{n}$ denotes the backward Euler convolution quadrature (CQ) approximation (with $\varphi^{j}=\varphi\left(t_{j}\right)$ ):

$$
\bar{\partial}_{\tau}^{\alpha} \varphi^{n}=\tau^{-\alpha} \sum_{j=0}^{n} b_{j}^{(\alpha)} \varphi^{n-j} \quad \text { with }(1-\xi)^{\alpha}=\sum_{j=0}^{\infty} b_{j}^{(\alpha)} \xi^{j} .
$$

Note that the weights $b_{j}^{(\alpha)}$ are given explicitly by $b_{j}^{(\alpha)}=(-1)^{j} \frac{\Gamma(\alpha+1)}{\Gamma(\alpha-j+1) \Gamma(j+1)}$, and thus

$$
b_{j}^{(\alpha)}=(-1)^{j} \frac{\alpha(\alpha-1) \cdots(\alpha-j+1)}{j !}, \quad j \geq 1,
$$

from which it can be verified directly that $b_{0}^{(\alpha)}=1$ and $b_{j}^{(\alpha)}<0$ for $j \geq 1$. Similarly, one deduces $b_{j}^{(\alpha-1)}>0$ for $j=0,1, \ldots$. Meanwhile, by definition, we have (with $\left.\varphi^{0}=0\right)$

$\bar{\partial}_{\tau}^{\alpha-1} \bar{\partial}_{\tau} \varphi^{n}=\tau^{-\alpha} \sum_{j=1}^{n} b_{n-j}^{(\alpha-1)}\left(\varphi^{j}-\varphi^{j-1}\right)=\tau^{-\alpha}\left(b_{0}^{(\alpha-1)} \varphi^{n}+\sum_{j=1}^{n-1}\left(b_{n-j}^{(\alpha-1)}-b_{n-j-1}^{(\alpha-1)}\right) \varphi^{j}\right)$.

Direct computation shows $b_{j}^{(\alpha-1)}-b_{j-1}^{(\alpha-1)}=b_{j}^{(\alpha)}$. This and the fact $b_{0}^{(\alpha-1)}=b_{0}^{(\alpha)}=1$ show the following associativity of convolution quadrature (with $\varphi^{0}=0$ )

$$
\bar{\partial}_{\tau}^{\alpha-1} \bar{\partial}_{\tau} \varphi^{n}=\bar{\partial}_{\tau}^{\alpha} \varphi^{n}
$$

Upon letting the discrete operator $A_{h}(q): X_{h} \rightarrow X_{h}$ by $-\left(A_{h}(q) v_{h}, \chi\right)=\left(q \nabla v_{h}, \nabla \chi\right)$ for all $v_{h}, \chi \in X_{h}$, the fully discrete scheme (3.3) can be rewritten as

$$
\bar{\partial}_{\tau}^{\alpha}\left(U_{h}^{n}-U_{h}^{0}\right)-A_{h}(q) U_{h}^{n}=P_{h} f^{n}, \quad n=1,2, \ldots, N .
$$

Now we can formulate the finite element discretization of problem (2.1)-(2.2):

$$
\min _{q_{h} \in \mathcal{A}_{h}} J_{\gamma, h, \tau}\left(q_{h}\right)=\frac{\tau}{2} \sum_{n=1}^{N} \int_{\Omega}\left|U_{h}^{n}\left(q_{h}\right)-z_{n}^{\delta}\right|^{2} \mathrm{~d} x+\frac{\gamma}{2}\left\|\nabla q_{h}\right\|_{L^{2}(\Omega)}^{2}
$$

Copyright (c) by SIAM. Unauthorized reproduction of this article is prohibited. 
with $z_{n}^{\delta}=\tau^{-1} \int_{t_{n-1}}^{t_{n}} z^{\delta} \mathrm{d} t$, and $U_{h}^{n}\left(q_{h}\right)$ satisfying $U_{h}^{0}\left(q_{h}\right)=P_{h} u_{0}$ and

$$
\bar{\partial}_{\tau}^{\alpha}\left(U_{h}^{n}\left(q_{h}\right)-U_{h}^{0}\right)-A_{h}\left(q_{h}\right) U_{h}^{n}\left(q_{h}\right)=P_{h} f^{n}, \quad n=1,2, \ldots, N .
$$

The discrete admissible set $\mathcal{A}_{h}$ is taken to be

$$
\mathcal{A}_{h}=\left\{q_{h} \in V_{h}: c_{0} \leq q_{h}(x) \leq c_{1} \quad \text { in } \Omega\right\} .
$$

Clearly, $\mathcal{A}_{h}=\mathcal{A} \cap V_{h}$. Problem (3.6)-(3.7) is a finite-dimensional nonlinear optimization problem with PDE and box constraint and can be solved efficiently. The analysis of problem (3.6)-(3.7) is the main focus of sections 3.2 and 4.

3.2. Existence and convergence. This part is devoted to the convergence analysis of the discrete approximations given by the scheme (3.6)-(3.7) to the continuous formulation (2.1)-(2.2). We begin with some a priori estimate on the solutions of the time stepping scheme (3.3). The proof relies on positivity of CQ.

Lemma 3.1. Let $V_{h}^{n} \in X_{h}$ solve

$$
\left(\bar{\partial}_{\tau}^{\alpha} V_{h}^{n}, \chi\right)+\left(q_{h} \nabla V_{h}^{n}, \nabla \chi\right)=\left(f_{h}^{n}, \chi\right) \quad \forall \chi \in X_{h}, \quad n=1,2, \ldots, N,
$$

with $V_{h}^{0}=0$. Then there holds

$$
\tau \sum_{n=1}^{N}\left(\nabla V_{h}^{n}, \nabla V_{h}^{n}\right) \leq c \tau \sum_{n=1}^{N}\left(f_{h}^{n}, V_{h}^{n}\right) .
$$

Proof. Letting $\chi=V_{h}^{n} \in X_{h}$ and then summing over $n$ leads to

$$
\tau \sum_{n=1}^{N}\left(\bar{\partial}_{\tau}^{\alpha} V_{h}^{n}, V_{h}^{n}\right)+\tau \sum_{n=1}^{N}\left(q_{h} \nabla V_{h}^{n}, \nabla V_{h}^{n}\right)=\tau \sum_{n=1}^{N}\left(f_{h}^{n}, V_{h}^{n}\right) .
$$

Now we shall show that the first term on the left-hand side is nonnegative. To this end, we extend $\left\{V_{h}^{n}\right\}_{n=0}^{N}$ to $\left\{V_{h}^{n}\right\}_{n=-\infty}^{n=\infty}$ and $\left\{b_{n}^{(\alpha)}\right\}_{n=0}^{n=\infty}$ to $\left\{b_{n}^{(\alpha)}\right\}_{n=-\infty}^{n=\infty}$ by zero. Then $\bar{\partial}_{\tau}^{\alpha} V_{h}^{n}$ can be written as $\bar{\partial}_{\tau}^{\alpha} V_{h}^{n}=\tau^{-\alpha} \sum_{j=-\infty}^{\infty} b_{n-j}^{(\alpha)} V_{h}^{j}$. Next we denote the discrete Fourier transform $\left[\widetilde{V_{h}^{n}}\right](\zeta)$ by $\left[\widetilde{V_{h}^{n}}\right](\zeta)=\sum_{n=-\infty}^{\infty} V_{h}^{n} e^{-\mathrm{i} n \zeta}$. By Parseval's theorem, since $V_{h}^{0}=0$, we have

$$
\sum_{j=1}^{N}\left(\bar{\partial}_{\tau}^{\alpha} V_{h}^{n}, V_{h}^{n}\right)=\frac{1}{2 \pi} \int_{-\pi}^{\pi}\left(\left[\widetilde{\bar{\partial}_{\tau}^{\alpha} V_{h}^{n}}\right](\zeta), \widetilde{\left[V_{h}^{n}\right]}(\zeta)^{*}\right) \mathrm{d} \zeta .
$$

By the property of discrete Fourier transform, we have

$$
\begin{aligned}
\sum_{j=1}^{N}\left(\bar{\partial}_{\tau}^{\alpha} V_{h}^{n}, V_{h}^{n}\right) & =\frac{\tau^{-\alpha}}{2 \pi} \int_{-\pi}^{\pi} \widetilde{\left[b_{n}^{(\alpha)}\right]}\left|\widetilde{\left[V_{h}^{n}\right]}(\zeta)\right|^{2} \mathrm{~d} \zeta \\
& =\frac{\tau^{-\alpha}}{\pi} \int_{0}^{\pi}\left[\Re\left(1-e^{-\mathrm{i} \zeta}\right)^{\alpha}\right]\left|\widetilde{\left[y_{n}\right]}(\zeta)\right|^{2} \mathrm{~d} \zeta \geq 0 .
\end{aligned}
$$

Then the Cauchy-Schwarz inequality and Poincaré's inequality imply the desired estimate. 
LEMma 3.2. The following statements hold:

$$
\sum_{n=0}^{m} b_{n}^{(\alpha)}=b_{m}^{(\alpha-1)} \quad \text { and } \quad\left|\tau^{-\alpha} \sum_{n=0}^{m} b_{n}^{(\alpha)}\right| \leq c t_{m+1}^{-\alpha} .
$$

Proof. Let $\sum_{n=0}^{m} b_{n}^{(\alpha)}=v_{m}$. Then by changing the order of summation, we have

$$
\begin{aligned}
\sum_{m=0}^{\infty} v_{m} \xi^{m} & =\sum_{m=0}^{\infty} \xi^{m} \sum_{n=0}^{m} b_{n}^{(\alpha)}=\sum_{n=0}^{\infty} b_{n}^{(\alpha)} \sum_{m=n}^{\infty} \xi^{m} \\
& =\sum_{n=0}^{\infty} b_{n}^{(\alpha)} \xi^{n} \sum_{m=n}^{\infty} \xi^{m-n}=\left(\sum_{n=0}^{\infty} b_{n}^{(\alpha)} \xi^{n}\right)\left(\sum_{m=0}^{\infty} \xi^{m}\right) \\
& =(1-\xi)^{\alpha}(1-\xi)^{-1}=(1-\xi)^{\alpha-1} .
\end{aligned}
$$

Therefore, $v_{m}=b_{m}^{(\alpha-1)} \leq c(m+1)^{-\alpha}[25$, Lemma 2.3], which shows the second assertion. [4]:

The next result gives a discrete analogue of the following well known inequality

$$
\varphi(t) \partial_{t}^{\alpha}(\varphi(t)-\varphi(0)) \geq \frac{1}{2} \partial_{t}^{\alpha}\left(|\varphi(t)|^{2}-\left.\varphi(0)\right|^{2}\right) .
$$

It is useful for deriving a priori estimates on the fully discrete solutions.

Lemma 3.3. Let $\bar{\partial}_{\tau}^{\alpha} \varphi^{n}$ be the backward Euler $C Q$ defined as (3.4). Then there holds

$$
\left(\bar{\partial}_{\tau}^{\alpha}\left(\varphi^{n}-\varphi^{0}\right)\right) \varphi^{n} \geq \frac{1}{2} \bar{\partial}_{\tau}^{\alpha}\left(\left|\varphi^{n}\right|^{2}-\left|\varphi^{0}\right|^{2}\right) .
$$

Proof. By the definition of backward Euler CQ in (3.4), we deduce

$$
\left(\bar{\partial}_{\tau}^{\alpha}\left(\varphi^{n}-\varphi^{0}\right)\right) \varphi^{n}=\tau^{-\alpha}\left(\left|\varphi^{n}\right|^{2}+\sum_{j=0}^{n-1} b_{n-j}^{(\alpha)} \varphi^{n} \varphi^{j}-\left(\sum_{j=0}^{n} b_{n-j}^{(\alpha)}\right) \varphi^{n} \varphi^{0}\right) .
$$

Now since the binomial coefficient $b_{j}^{(\alpha)}<0$ for $j \geq 1$, we deduce

$$
\sum_{j=0}^{n-1} b_{n-j}^{(\alpha)} \varphi^{n} \varphi^{j} \geq \frac{1}{2} \sum_{j=0}^{n-1} b_{n-j}^{(\alpha)}\left|\varphi^{n}\right|^{2}+\frac{1}{2} \sum_{j=0}^{n-1} b_{n-j}^{(\alpha)}\left|\varphi^{j}\right|^{2}
$$

and

$$
\left(\sum_{j=0}^{n} b_{n-j}^{(\alpha)}\right) \varphi^{n} \varphi^{0} \leq \frac{1}{2}\left(\sum_{j=0}^{n} b_{n-j}^{(\alpha)}\right)\left|\varphi^{n}\right|^{2}+\frac{1}{2}\left(\sum_{j=0}^{n} b_{n-j}^{(\alpha)}\right)\left|\varphi^{0}\right|^{2} .
$$

Then the desired result follows immediately.

The next result gives a discrete continuity result. Then

Lemma 3.4. Let the sequence $\left\{q_{h}^{j}\right\} \subset \mathcal{A}_{h}$ be convergent to $q_{h}^{*} \in \mathcal{A}_{h}$ in $L^{1}(\Omega)$.

$$
\lim _{j \rightarrow \infty} \tau \sum_{n=1}^{N} \int_{\Omega}\left|U_{h, \tau}^{n}\left(q_{h}^{j}\right)-z_{n}^{\delta}\right|^{2} \mathrm{~d} x=\tau \sum_{n=1}^{N} \int_{\Omega}\left|U_{h, \tau}^{n}\left(q_{h}^{*}\right)-z_{n}^{\delta}\right|^{2} \mathrm{~d} x .
$$

Copyright $@$ by SIAM. Unauthorized reproduction of this article is prohibited. 
Proof. Using Lemma 3.1, the proof is similar to that of Lemma 2.3, since in a finite-dimensional space $V_{h}$, all norms are equivalent, and the convergence in $L^{1}(\Omega)$ implies almost every convergence [16]. Thus the proof is omitted.

Then we can obtain the existence of a discrete minimizer $q_{h}^{*} \in \mathcal{A}_{h}$. The proof is identical to that in Theorem 2.4, and hence omitted. Note that the discrete minimizer $q_{h}^{*}$ depends implicitly also on the time step size $\tau$ through the weak formulation (3.7).

Theorem 3.5. Under Assumption 2.1, there exists one minimizer $q_{h}^{*} \in \mathcal{A}_{h}$ to (3.6)-(3.7)

Below we analyze the convergence of the sequence $\left\{q_{h}^{*}\right\}_{h>0}$ as $h, \tau \rightarrow 0$. The next result is an analogue of Lemma 2.3 and plays an important role in the convergence analysis. For the sequence of discrete solutions $U_{h, \tau}^{n} \equiv U_{h, \tau}^{n}\left(q_{h}\right) \in X_{h}$ to problem (3.7), we define a piecewise constant in time interpolation $u_{h, \tau}(t)$ by

$$
u_{h, \tau}(t)=U_{h, \tau}^{n}, \quad t \in\left[t_{n}, t_{n+1}\right), \quad n=0, \ldots, N-1 .
$$

Lemma 3.6. Let $U_{h, \tau}^{n} \equiv U_{h, \tau}^{n}\left(q_{h}\right) \in X_{h}$ be the discrete solutions to problem (3.7) with $q_{h} \in \mathcal{A}_{h}$, and the sequence $\left\{q_{h} \in \mathcal{A}_{h}\right\}_{h>0}$ convergent to some $q^{*} \in \mathcal{A}$ a.e. as $h, \tau \rightarrow 0^{+}$. Then under Assumption 2.1, for the piecewise constant interpolation $u_{h, \tau}$ defined in (3.8), there holds

$$
u_{h, \tau}\left(q_{h}\right) \rightarrow u\left(q^{*}\right) \text { weakly in } L^{2}\left(0, T ; H^{1}(\Omega)\right), \quad \text { as } h, \tau \rightarrow 0 .
$$

Proof. Taking the test function $\chi=U_{h}^{n}-U_{h}^{0}$ in (3.7) and summing over $n$ yields

$$
\tau \sum_{n=0}^{N}\left(\bar{\partial}_{\tau}^{\alpha}\left(U_{h}^{n}-U_{h}^{0}\right), U_{h}^{n}-U_{h}^{0}\right)+\tau \sum_{n=1}^{N}\left(q_{h} \nabla U_{h}^{n}, \nabla\left(U_{h}^{n}-U_{h}^{0}\right)\right)=\tau \sum_{n=1}^{n}\left(f_{h}^{n}, U_{h}^{n}-U_{h}^{0}\right) .
$$

This identity, the nonnegativity of the discrete convolution $\bar{\partial}_{\tau}^{\alpha}$ (see the proof of Lemma 3.1), the Poincaré inequality and Young's inequality, and the $L^{2}(\Omega)$ stability of $P_{h}$ lead to

$$
\begin{aligned}
\tau \sum_{n=1}^{N}\left\|\nabla U_{h}^{n}\right\|_{L^{2}(\Omega)}^{2} & \leq c \tau \sum_{n=1}^{N}\left(\left\|\nabla U_{h}^{0}\right\|_{L^{2}(\Omega)}^{2}+\left\|f_{h}^{n}\right\|_{H^{-1}(\Omega)}^{2}\right) \\
& \leq c\left(\left\|\nabla u_{0}\right\|_{L^{2}(\Omega)}^{2}+\|f\|_{L^{2}\left(0, T ; H^{-1}(\Omega)\right)}^{2}\right) .
\end{aligned}
$$

Thus, the sequence $\left\{u_{h, \tau}\right\}_{h, \tau>0}$ is uniformly bounded in $L^{2}\left(0, T ; H^{1}(\Omega)\right)$, and thus there exists a subsequence, still denoted by $\left\{u_{h, \tau}\right\}_{h, \tau>0}$, and some $u^{*} \in L^{2}\left(0, T ; H^{1}(\Omega)\right)$ such that

$$
u_{h, \tau} \text { converges weakly to } u^{*} \text { in } L^{2}\left(0, T ; H^{1}(\Omega)\right) .
$$

Meanwhile, by taking the test function $\chi=\bar{\partial}_{\tau}^{\alpha}\left(U_{h}^{n}-U_{h}^{0}\right)$ in (3.7),

$$
\begin{aligned}
& \tau \sum_{n=0}^{N}\left(\bar{\partial}_{\tau}^{\alpha}\left(U_{h}^{n}-U_{h}^{0}\right), \bar{\partial}_{\tau}^{\alpha}\left(U_{h}^{n}-U_{h}^{0}\right)\right)+\tau \sum_{n=1}^{N}\left(q_{h} \nabla U_{h}^{n}, \bar{\partial}_{\tau}^{\alpha} \nabla\left(U_{h}^{n}-U_{h}^{0}\right)\right) \\
& \quad=\tau \sum_{n=1}^{n}\left(f_{h}^{n}, \bar{\partial}_{\tau}^{\alpha}\left(U_{h}^{n}-U_{h}^{0}\right)\right) .
\end{aligned}
$$

Copyright $@$ by SIAM. Unauthorized reproduction of this article is prohibited. 
Then Lemma 3.3, the fact that $b_{j}^{(\alpha-1)}>0$ for all $j \geq 0$, and Lemma 3.2 lead to

$$
\begin{aligned}
2 \tau & \sum_{n=1}^{N}\left(\nabla U_{h}^{n}\right)^{\mathrm{t}} \bar{\partial}_{\tau}^{\alpha} \nabla\left(U_{h}^{n}-U_{h}^{0}\right) \geq \tau \sum_{n=1}^{N} \bar{\partial}_{\tau}^{\alpha}\left(\left\|\nabla U_{h}^{n}\right\|_{L^{2}(\Omega)}^{2}-\left\|\nabla U_{h}^{0}\right\|_{L^{2}(\Omega)}^{2}\right) \\
& =\tau \sum_{j=0}^{N}\left(\left\|\nabla U_{h}^{j}\right\|_{L^{2}(\Omega)}^{2}-\left\|\nabla U_{h}^{0}\right\|_{L^{2}(\Omega)}^{2}\right) b_{N-j}^{(\alpha-1)} \\
& \geq \tau \sum_{j=0}^{N}-\left\|\nabla U_{h}^{0}\right\|_{L^{2}(\Omega)}^{2} b_{N-j}^{(\alpha-1)} \geq-c\left\|\nabla U_{h}^{0}\right\|_{L^{2}(\Omega)}^{2} .
\end{aligned}
$$

Hence, there holds $\tau \sum_{n=1}^{N}\left(q_{h} \nabla U_{h}^{n}, \bar{\partial}_{\tau}^{\alpha} \nabla\left(U_{h}^{n}-U_{h}^{0}\right)\right) \geq-c\left\|\nabla U_{h}^{0}\right\|_{L^{2}(\Omega)}^{2}$. This and Young's inequality imply

$$
\tau \sum_{n=1}^{N}\left\|\bar{\partial}_{\tau}^{\alpha}\left(U_{h}^{n}-U_{h}^{0}\right)\right\|_{L^{2}(\Omega)}^{2} \leq c\left(\left\|\nabla u_{0}\right\|_{L^{2}(\Omega)}^{2}+\|f\|_{L^{2}\left(0, T ; L^{2}(\Omega)\right)}^{2}\right) .
$$

Thus the sequence of piecewise constant interpolation, denoted by $\left\{\bar{\partial}_{\tau}^{\alpha}\left(u_{h, \tau}-U_{h}^{0}\right)\right\}_{h, \tau>0}$, is uniformly bounded in $L^{2}\left(0, T ; L^{2}(\Omega)\right)$, and there exists a subsequence, still denoted by $\left\{\bar{\partial}_{\tau}^{\alpha}\left(u_{h, \tau}-U_{h}^{0}\right)\right\}_{h, \tau>0}$, and some $v^{*} \in L^{2}\left(0, T ; L^{2}(\Omega)\right)$ such that it converges to $v^{*}$ weakly in $L^{2}\left(0, T ; L^{2}(\Omega)\right)$. Next we claim that $u^{*}$ satisfies the weak formulation of $u\left(q^{*}\right)$ (cf. (2.2)). To this end, we take a smooth test function $\phi \in C^{1}\left([0, T] ; \dot{H}^{1}(\Omega)\right)$ with $\phi(T)=0$ and define an approximation $\phi_{h, \tau}$ by $\phi_{h, \tau}(t)=\tau^{-1} \int_{t_{n-1}}^{t_{n}} P_{h} \phi(t) \mathrm{d} t$, $t \in\left(t_{n-1}, t_{n}\right]$. Then the density of $X_{h}$ in $\dot{H}^{1}(\Omega)$ and piecewise constant functions in $L^{2}(0, T)$ imply that $\lim _{h, \tau \rightarrow 0^{+}}\left\|\phi_{h, \tau}-\phi\right\|_{L^{2}\left(0, T ; H^{1}(\Omega)\right)}=0$. Hence, by discrete summation by parts and straightforward computation, there holds

$$
\begin{aligned}
\tau \sum_{n=1}^{N}\left(\bar{\partial}_{\tau}^{\alpha}\left(U_{h}^{n}-U_{h}^{0}\right), \phi_{h, \tau}\left(t_{n}\right)\right) & =\left(\bar{\partial}_{\tau}^{\alpha}\left(u_{h, \tau}-U_{h}^{0}\right), P_{h} \phi(t)\right)_{L^{2}\left(0, T ; L^{2}(\Omega)\right)} \\
& =\left(u_{h, \tau}-U_{h}^{0},{ }^{R} \bar{\partial}_{\tau}^{\alpha} P_{h} \phi(t)\right)_{L^{2}\left(0, T ; L^{2}(\Omega)\right)}
\end{aligned}
$$

where the notation ${ }^{R} \bar{\partial}_{\tau}^{\alpha} P_{h} \phi(t)$ denotes ${ }^{R} \bar{\partial}_{\tau}^{\alpha} P_{h} \phi(t)=\sum_{i=n}^{N} b_{n-i}^{(\alpha)} P_{h} \phi(t+(i-n) \tau)$ for $t \in\left(t_{n-1}, t_{n}\right], n=1,2, \ldots, N$. By the approximation property of ${ }^{R} \bar{\partial}_{\tau}^{\alpha}$ and $P_{h}$ (see, e.g., $\left[45\right.$, section 2.2]), since $\phi \in C^{1}\left([0, T] ; \dot{H}^{1}(\Omega)\right),{ }^{R} \bar{\partial}_{\tau}^{\alpha} P_{h} \phi(t)$ converges to ${ }^{R} \partial_{t}^{\alpha} \phi(t)$ in $L^{2}\left(0, T ; L^{2}(\Omega)\right)$ as $h, \tau \rightarrow 0^{+}$, and

$$
\lim _{h, \tau \rightarrow 0}\left(u_{h, \tau}-U_{h}^{0},{ }^{R} \bar{\partial}_{\tau}^{\alpha} P_{h} \phi(t)\right)_{L^{2}\left(0, T ; L^{2}(\Omega)\right)}=\left(u^{*}-u_{0},{ }^{R} \partial_{t}^{\alpha} \phi(t)\right)_{L^{2}\left(0, T ; L^{2}(\Omega)\right)},
$$

and meanwhile, by the weak convergence of $\bar{\partial}_{\tau}^{\alpha}\left(u_{h, \tau}-U_{h}^{0}\right)$ to $v^{*}$ in $L^{2}\left(0, T ; L^{2}(\Omega)\right)$ and the approximation property of $P_{h}$,

$$
\lim _{h, \tau \rightarrow 0}\left(\bar{\partial}_{\tau}^{\alpha}\left(u_{h, \tau}-U_{h}^{0}\right), P_{h} \phi(t)\right)_{L^{2}\left(0, T ; L^{2}(\Omega)\right)}=\left(v^{*}, \phi(t)\right)_{L^{2}\left(0, T ; L^{2}(\Omega)\right)} .
$$

Comparing the preceding two identities shows that $v^{*}=\partial_{t}^{\alpha}\left(u^{*}-u_{0}\right)$, i.e., $v^{*}$ is the weak fractional order derivative of $u^{*}-u_{0}$. Now taking the test function $\chi=\phi_{h, \tau}\left(t_{n}\right)$

Copyright $@$ by SIAM. Unauthorized reproduction of this article is prohibited. 
in (3.7) and summing over $n$, we obtain

$$
\begin{aligned}
\tau \sum_{n=0}^{N} & \left(\bar{\partial}_{\tau}^{\alpha}\left(U_{h}^{n}-U_{h}^{0}\right), \phi_{h, \tau}\left(t_{n}\right)\right)+\tau \sum_{n=1}^{N}\left(q_{h} \nabla U_{h}^{n}, \nabla \phi_{h, \tau}\left(t_{n}\right)\right) \\
& =\tau \sum_{n=1}^{N}\left(f_{h}^{n}, \phi_{h, \tau}\left(t_{n}\right)\right)
\end{aligned}
$$

and by the definition of piecewise constant interpolations $\bar{\partial}_{\tau}\left(U_{h, \tau}^{n}-U_{h}^{0}\right)$ and $u_{h, \tau}(t)$ and the construction of the test function $\phi_{h, \tau}\left(t_{n}\right)$, it is equivalent to

$$
\begin{aligned}
& \left(\bar{\partial}_{\tau}^{\alpha}\left(u_{h, \tau}^{n}-U_{h}^{0}\right), P_{h} \phi\right)_{L^{2}\left(0, T ; L^{2}(\Omega)\right)}+\left(q_{h} \nabla u_{h, \tau}, \nabla P_{h} \phi(t)\right)_{L^{2}\left(0, T ; L^{2}(\Omega)\right)} \\
& \quad=\left(f_{h, \tau}, P_{h} \phi(t)\right)_{L^{2}\left(0, T ; L^{2}(\Omega)\right)}
\end{aligned}
$$

where $f_{h, \tau}(t)=\tau^{-1} \int_{t_{n-1}}^{t_{n}} P_{h} f(t) \mathrm{d} t$ for $t \in\left(t_{n-1}, t_{n}\right], n=1, \ldots, N$, for which there holds $\lim _{h, \tau \rightarrow 0^{+}}\left\|f_{h, \tau}-f\right\|_{L^{2}\left(0, T ; L^{2}(\Omega)\right)}=0$. Upon passing the limit on both sides, we deduce

$$
\begin{aligned}
\lim _{h, \tau \rightarrow 0^{+}}\left(\bar{\partial}_{\tau}^{\alpha}\left(U_{h, \tau}^{n}-U_{h}^{0}\right), P_{h} \phi\right)_{L^{2}\left(0, T ; L^{2}(\Omega)\right)} & =\left(\partial_{t}^{\alpha}\left(u^{*}-u_{0}\right), \phi\right)_{L^{2}\left(0, T ; L^{2}(\Omega)\right)} \\
\lim _{h, \tau \rightarrow 0^{+}}\left(f_{h, \tau}, P_{h} \phi(t)\right)_{L^{2}\left(0, T ; L^{2}(\Omega)\right)} & =(f, \phi)_{L^{2}\left(0, T ; L^{2}(\Omega)\right)} .
\end{aligned}
$$

Further, to analyze the term $\left(q_{h} \nabla u_{h, \tau}, \nabla P_{h} \phi(t)\right)_{L^{2}\left(0, T ; L^{2}(\Omega)\right)}$, we employ the following splitting:

$$
\begin{aligned}
&\left|\left(q_{h} \nabla u_{h, \tau}, \nabla P_{h} \phi(t)\right)_{L^{2}\left(0, T ; L^{2}(\Omega)\right)}-\left(q^{*} \nabla u^{*}, \nabla \phi(t)\right)_{L^{2}\left(0, T ; L^{2}(\Omega)\right)}\right| \\
& \leq\left|\left(q_{h} \nabla u_{h, \tau}, \nabla P_{h} \phi(t)\right)_{L^{2}\left(0, T ; L^{2}(\Omega)\right)}-\left(q_{h} \nabla u_{h, \tau}, \nabla \phi(t)\right)_{L^{2}\left(0, T ; L^{2}(\Omega)\right)}\right| \\
&+\left|\left(q_{h} \nabla u_{h, \tau}, \nabla \phi(t)\right)_{L^{2}\left(0, T ; L^{2}(\Omega)\right)}-\left(q^{*} \nabla u_{h, \tau}, \nabla \phi(t)\right)_{L^{2}\left(0, T ; L^{2}(\Omega)\right)}\right| \\
&+\left|\left(q^{*} \nabla u_{h, \tau}, \nabla \phi(t)\right)_{L^{2}\left(0, T ; L^{2}(\Omega)\right)}-\left(q^{*} \nabla u^{*}, \nabla \phi(t)\right)_{L^{2}\left(0, T ; L^{2}(\Omega)\right)}\right|:=\mathrm{I}+\mathrm{II}+\mathrm{III} .
\end{aligned}
$$

We bound the three terms separately. By the approximation property of $P_{h}$ and uniform boundedness of $u_{h, \tau}$ in $L^{2}\left(0, T ; H^{1}(\Omega)\right)$ due to (3.9), we deduce

$$
\lim _{h, \tau \rightarrow 0^{+}} \mathrm{I} \leq \lim _{h, \tau \rightarrow 0^{+}} c\left\|u_{h, \tau}\right\|_{L^{2}\left(0, T ; H^{1}(\Omega)\right)}\left\|P_{h} \phi-\phi\right\|_{L^{2}\left(0, T ; H^{1}(\Omega)\right)}=0 .
$$

Next, since $q_{h}$ converges to $q^{*}$ a.e. and (3.9), by the dominated convergence theorem [16, Theorem 1.9] (with the argument in Lemma 2.3), we have

$$
\lim _{h, \tau \rightarrow 0^{+}} \mathrm{II} \leq \lim _{h, \tau \rightarrow 0^{+}}\left\|u_{h, \tau}\right\|_{L^{2}\left(0, T ; H^{1}(\Omega)\right)}\left\|\left(q_{h}-q^{*}\right) \phi\right\|_{L^{2}\left(0, T ; H^{1}(\Omega)\right)}=0 .
$$

The third term III tends to zero as $h, \tau \rightarrow 0^{+}$, in view of the weak convergence in (3.9). Consequently, combining the three assertions together yields

$$
\lim _{h, \tau \rightarrow 0^{+}}\left(q_{h} \nabla u_{h, \tau}, \nabla P_{h} \phi(t)\right)_{L^{2}\left(0, T ; L^{2}(\Omega)\right)}=\left(q^{*} \nabla u^{*}, \nabla \phi(t)\right)_{L^{2}\left(0, T ; L^{2}(\Omega)\right)} .
$$

In sum, the limit $u^{*}$ satisfies that for any $\phi \in C^{1}\left([0, T] ; \dot{H}^{1}(\Omega)\right)$, there holds

$$
\left(\partial_{t}^{\alpha}\left(u^{*}-u_{0}\right), \phi\right)_{L^{2}\left(0, T ; L^{2}(\Omega)\right)}+\left(q^{*} \nabla u^{*}, \nabla \phi\right)_{L^{2}\left(0, T ; L^{2}(\Omega)\right)}=(f, \phi)_{L^{2}\left(0, T ; L^{2}(\Omega)\right)} .
$$

Copyright (c) by SIAM. Unauthorized reproduction of this article is prohibited. 
By the density of the space $C^{1}\left([0, T] ; \dot{H}^{1}(\Omega)\right)$ in $L^{2}\left(0, T ; \dot{H}^{1}(\Omega)\right)$, the identity holds also for any $\phi \in L^{2}\left(0, T ; \dot{H}^{1}(\Omega)\right)$. This immediately shows that $u^{*}$ is a weak solution to problem (1.1) with $q^{*}$, i.e., $u^{*}=u\left(q^{*}\right)$. Since every subsequence contains a convergent sub-subsequence, the whole sequence converges to $u\left(q^{*}\right)$. This completes the proof of the lemma.

Now we can state the main result of this part, i.e., the convergence of the discrete solutions $\left\{q_{h}^{*}\right\}_{h>0}$ to the continuous optimization problem (2.1)-(2.2). With Lemma 3.6 at hand, the proof is standard and it is included only for completeness.

THEOREM 3.7. Let $\left\{q_{h}^{*}\right\}_{h>0}$ be a sequence of minimizers to problem (3.6)-(3.7). Then under Assumption 2.1, it contains a subsequence convergent to a minimizer of problem $(2.1)-(2.2)$ in $H^{1}(\Omega)$.

Proof. Since the constant function $q_{h} \equiv c_{0}$ belongs to the admissible set $\mathcal{A}_{h}$ for any $h$, there holds $J_{\gamma, h, \tau}\left(q_{h}^{*}\right) \leq J_{\gamma, h, \tau}\left(c_{0}\right)<\infty$, from which it directly follows that the sequence $\left\{q_{h}^{*}\right\}_{h>0}$ is uniformly bounded in the $H^{1}(\Omega)$-seminorm. This and the box constraint in $\mathcal{A}_{h}$ imply that the sequence $\left\{q_{h}^{*} \in \mathcal{A}_{h}\right\}_{h>0}$ is uniformly bounded in the $H^{1}(\Omega)$ norm. Thus there exists a subsequence, still denoted by $\left\{q_{h}^{*}\right\}_{h>0}$, such that it converges weakly in the $H^{1}(\Omega)$ to some $q^{*} \in \mathcal{A}$. We claim that $q^{*}$ is a minimizer to problem (2.1)-(2.2). For any $q \in \mathcal{A}$, by the density of $W^{1, \infty}(\Omega)$ in $H^{1}(\Omega)$ [16] (e.g., by means of mollifier), there exists a sequence $\left\{q^{\epsilon}\right\}_{\epsilon>0} \subset \mathcal{A} \cap W^{1, \infty}(\Omega)$ such that $\lim _{\epsilon \rightarrow 0^{+}}\left\|q^{\epsilon}-q\right\|_{H^{1}(\Omega)}=0$ and almost everywhere. Now let $q_{h}^{\epsilon}=\mathcal{I}_{h} q^{\epsilon} \in V_{h}$. By the minimizing property of $q_{h}^{*}$, there holds

$$
J_{\gamma, h, \tau}\left(q_{h}^{*}\right) \leq J_{\gamma, h, \tau}\left(q_{h}^{\epsilon}\right) .
$$

By the weak lower semicontinuity of norms, we have

$$
\left\|\nabla q^{*}\right\|_{L^{2}(\Omega)} \leq \liminf _{h \rightarrow 0}\left\|\nabla q_{h}^{*}\right\|_{L^{2}(\Omega)} .
$$

Similarly, by the weak convergence of $u_{h, \tau}\left(q_{h}^{*}\right)$ to $u\left(q^{*}\right)$ in $L^{2}\left(0, T ; H^{1}(\Omega)\right)$ in Lemma 3.6 and the embedding $H^{1}(\Omega) \hookrightarrow L^{2}(\Omega)$, and the construction of the function $z_{\tau}^{\delta}(t)=\tau^{-1} \int_{t_{n-1}}^{t_{n}} z^{\delta}(t) \mathrm{d} t$, for $t \in\left(t_{n-1}, t_{n}\right], n=1, \ldots, N, \lim _{\tau \rightarrow 0^{+}}\left\|z^{\delta}-z_{\tau}^{\delta}\right\|_{L^{2}\left(0, T ; L^{2}(\Omega)\right)}$ $=0$, we have $\left\|u\left(q^{*}\right)-z^{\delta}\right\|_{L^{2}\left(0, T ; L^{2}(\Omega)\right)}^{2} \leq \liminf _{h, \tau \rightarrow 0^{+}}\left\|u_{h, \tau}\left(q_{h}^{*}\right)-z_{\tau}^{\delta}\right\|_{L^{2}\left(0, T ; L^{2}(\Omega)\right)}=$ $\liminf \operatorname{in}_{h, \tau \rightarrow 0^{+}} \tau \sum_{n=1}^{N}\left\|U_{h}^{n}\left(q_{h}^{*}\right)-z_{n}^{\delta}\right\|_{L^{2}(\Omega)}^{2}$, and thus

$$
J_{\gamma}\left(q^{*}\right) \leq \lim _{h, \tau \rightarrow 0^{+}} J_{\gamma, h, \tau}\left(q_{h}^{*}\right) .
$$

Meanwhile, by Lemma 3.6 and the approximation property of the operator $\mathcal{I}_{h}$ in (3.2),

$$
\lim _{h, \tau \rightarrow 0^{+}} J_{\gamma, h, \tau}\left(q_{h}^{\epsilon}\right)=J_{\gamma}\left(q^{\epsilon}\right) .
$$

Thus, taking the limit as $h, \tau \rightarrow 0^{+}$in the inequality (3.11) yields $J_{\gamma}\left(q^{*}\right) \leq J_{\gamma}\left(q^{\epsilon}\right)$. Further, since $q^{\epsilon} \rightarrow q$ in $H^{1}(\Omega)$ and almost everywhere as $\epsilon \rightarrow 0^{+}$, by Lemma 2.3, there holds

$$
\lim _{\epsilon \rightarrow 0^{+}} J_{\gamma}\left(q^{\epsilon}\right)=J_{\gamma}(q) .
$$

Combining the relations (3.12)-(3.14) yields $J_{\gamma}\left(q^{*}\right) \leq J_{\gamma}(q)$ for any $q \in \mathcal{A}$. This shows the weak convergence to a minimizer $q^{*}$ in $H^{1}(\Omega)$. Meanwhile, by the weak lower semicontinuity of the norms and a standard argument by contradiction [20], we have $\lim _{h, \tau \rightarrow 0^{+}}\left\|\nabla q_{h}^{*}\right\|_{L^{2}(\Omega)}^{2}=\left\|\nabla q^{*}\right\|_{L^{2}(\Omega)}^{2}$. Hence, the subsequence $\left\{q_{h}^{*}\right\}_{h>0}$ converges to $q^{*}$ in $H^{1}(\Omega)$. This completes the proof of the theorem. 
Remark 3.8. Note that the continuity results in Lemmas 2.3 and 3.6 are stated with respect to almost everywhere convergence (deduced from the $L^{1}(\Omega)$ convergence of the sequence of the diffusion coefficient), which can be induced by other penalties with the underlying space compactly embedding into the space $L^{1}(\Omega)$, including the space of bounded variation [16]. Thus, upon minor modifications, the results in sections 2 and 3 hold also for related regularized formulations, e.g., total variation penalty, which is suitable for recovering discontinuous coefficients; see, e.g., [17, 7] for relevant studies in the parabolic and elliptic cases. Also note that the terminal observation $u(T)$ may require a stronger regularity condition on the source $f$ than Assumption 2.1 to ensure $u(q) \in C\left([0, T] ; L^{2}(\Omega)\right)$, depending on the value of the fractional order $\alpha$.

Remark 3.9. Due to the nonlinearity of the parameter-to-state map $q \mapsto u(q)$, the regularized output least-squares problem $(2.1)-(2.2)$ is expected to be highly nonconvex. Hence, numerically one can generally only guarantee to reach a stationary point $\hat{q}_{h}$ of the optimality system (OS) when solving the discrete optimization problem (3.6)-(3.7). One important theoretical question is the convergence of the sequence $\left\{\hat{q}_{h}\right\}_{h>0}$ of discrete stationary points for OS. Note that the convergence analysis in section 3 relies essentially on extracting a convergent subsequence of discrete minimizers $\left\{q_{h}^{*}\right\}_{h>0}$ in $L^{1}(\Omega)$, which in turn follows from the uniform a priori bound on $\left\{q_{h}^{*}\right\}_{h>0}$ in $H^{1}(\Omega)$, induced by the $H^{1}(\Omega)$-seminorm penalty. Thus, one crucial step in extending the analysis to stationary points is to derive a suitable uniform a priori bound on $\left\{\hat{q}_{h}\right\}_{h>0}$. This might be derived from the OS as follows. Indeed, the box constraints in the admissible set $\mathcal{A}_{h}$ allow bounding the discrete state $U_{h}^{n}\left(\hat{q}_{h}\right)$ (and thus also the discrete adjoint) uniformly in the discrete $L^{2}\left(0, T ; H^{1}(\Omega)\right)$ norm, and then the discrete variational inequality for $\hat{q}_{h}$ in OS allows uniformly bounding $\hat{q}_{h}$ in suitable Sobolev norm using "elliptic" regularity theory. We shall refrain from a detailed derivation of OS and the associated convergence analysis for stationary points, since the analysis in section 4 crucially exploits the minimizing property of the discrete minimizer and does not extend to stationary points directly.

4. Error estimates. Now we derive error estimates of approximations $q_{h}^{*}$ under the following regularity on the problem data.

Assumption 4.1. The following conditions hold.

(i) $u_{0} \in \dot{H}^{2}(\Omega), f \in C^{2}\left([0, T] ; L^{2}(\Omega)\right) \cap L^{\infty}\left(0, T ; \dot{H}^{\beta}(\Omega)\right)$ with $\beta>\max \left(\frac{d}{2}-1,0\right)$, and exact diffusion coefficient $q^{\dagger} \in W^{2, \infty}(\Omega)$.

(ii) $z^{\delta} \in C\left([0, T] ; L^{2}(\Omega)\right) \cap C^{2}\left((0, T] ; L^{2}(\Omega)\right)$ with

$$
t^{1-\alpha}\left\|z^{\delta \prime}(t)\right\|_{L^{2}(\Omega)}+t^{2-\alpha}\left\|z^{\delta \prime \prime}(t)\right\|_{L^{2}(\Omega)} \leq c .
$$

Under Assumption 4.1(i), there exists a unique solution $u \in C\left([0, T] ; \dot{H}^{2}(\Omega)\right) \cap$ $C^{2}\left((0, T] ; L^{2}(\Omega)\right)$ and for any $s \in[0, \beta)$ and $r \in[0,2]$, there holds

$$
\text { (4.1) }\|u(t)\|_{\dot{H}^{2}(\Omega)}+t^{\frac{s}{2} \alpha}\|u(t)\|_{\dot{H}^{2+s}(\Omega)}+t^{1-\left(1-\frac{s}{2}\right) \alpha}\left\|u^{\prime}(t)\right\|_{\dot{H}^{s}(\Omega)}+t^{2-\alpha}\left\|u^{\prime \prime}(t)\right\|_{L^{2}(\Omega)} \leq c .
$$

See $[47,23]$ for a proof of the regularity estimate.

The better temporal regularity on the observation $z^{\delta}$ and $u(q)$ enables slightly modifying the discrete optimization problem $J_{h, \tau, \gamma}$, instead of using

$$
z_{n}^{\delta}:=\tau^{-1} \int_{t_{n-1}}^{t_{n}} z^{\delta}(t) \mathrm{d} t
$$

In particular, we can employ the trapezoid rule: with $a_{0}=a_{N}=1 / 2$ and $a_{i}=1$, 
$i=1, \ldots, N-1$,

$$
\min _{q_{h} \in \mathcal{A}_{h}} J_{\gamma, h, \tau}\left(q_{h}\right)=\frac{\tau}{2} \sum_{n=0}^{N} a_{i} \int_{\Omega}\left|U_{h}^{n}\left(q_{h}\right)-z^{\delta}\left(t_{n}\right)\right|^{2} \mathrm{~d} x+\frac{\gamma}{2}\left\|\nabla q_{h}\right\|_{L^{2}(\Omega)}^{2},
$$

subject to $q_{h} \in \mathcal{A}_{h}$ and $U_{h}^{n}\left(q_{h}\right)$ satisfying $U_{h}^{0}=P_{h} u_{0}$ and

$$
\bar{\partial}_{\tau}^{\alpha}\left(U_{h}^{n}\left(q_{h}\right)-U_{h}^{0}\right)-A_{h}\left(q_{h}\right) U_{h}^{n}\left(q_{h}\right)=P_{h} f\left(t_{n}\right), \quad n=1,2, \ldots, N .
$$

This change allows deriving a better rate in $\tau$ in Theorem 4.8 below. Under Assumption 4.1, Theorems 3.5 and 3.7 in section 3 remain valid for problem (4.2)-(4.3). The goal of this part is to derive error estimates for the approximation constructed by (4.2)-(4.3).

We begin with some preliminary estimates under Assumption 4.1(i).

Lemma 4.2. Let $q^{\dagger}$ be the exact diffusion coefficient and $u \equiv u\left(q^{\dagger}\right)$ be the solution to problem (2.2), and $\left\{U_{h}^{n}\left(q^{\dagger}\right)\right\}$ and $\left\{U_{h}^{n}\left(\mathcal{I}_{h} q^{\dagger}\right)\right\}$ be the solutions to the scheme (3.3) corresponding to $q^{\dagger}$ and $\mathcal{I}_{h} q^{\dagger}$, respectively. Then under Assumption 4.1(i) with $\ell_{h}=$ $|\log h|$

$$
\begin{aligned}
\left\|u\left(t_{n}\right)-U_{h}^{n}\left(q^{\dagger}\right)\right\|_{L^{2}(\Omega)} & \leq c\left(\tau t_{n}^{\alpha-1}+h^{2} \ell_{h}\right), \\
\left\|u\left(t_{n}\right)-U_{h}^{n}\left(\mathcal{I}_{h} q^{\dagger}\right)\right\|_{L^{2}(\Omega)} & \leq c\left(\tau t_{n}^{\alpha-1}+h^{2} \ell_{h}\right) .
\end{aligned}
$$

Proof. The first estimate is immediate from [22]

$$
\begin{aligned}
\left\|u\left(t_{n}\right)-U_{h}^{n}\left(q^{\dagger}\right)\right\|_{L^{2}(\Omega)} \leq & c h^{2} \ell_{h}\left(\left\|A\left(q^{\dagger}\right) u_{0}\right\|_{L^{2}(\Omega)}+\|f\|_{L^{\infty}\left(0, T ; \dot{H}^{\beta}(\Omega)\right)}\right) \\
& +c \tau\left(t_{n}^{\alpha-1}\left\|A\left(q^{\dagger}\right) u_{0}+f(0)\right\|_{L^{2}(\Omega)}\right. \\
& \left.+\int_{0}^{t_{n}}\left(t_{n}-s\right)^{\alpha-1}\left\|f^{\prime}(s)\right\|_{L^{2}(\Omega)} \mathrm{d} s\right) .
\end{aligned}
$$

To show the second estimate, we bound $\rho_{h}^{n}:=U_{h}^{n}\left(q^{\dagger}\right)-U_{h}^{n}\left(\mathcal{I}_{h} q^{\dagger}\right)$, which satisfies $\rho_{h}^{0}=0$ and

$$
\bar{\partial}_{\tau}^{\alpha} \rho_{h}^{n}-A_{h}\left(q^{\dagger}\right) \rho_{h}^{n}=\left[A_{h}\left(q^{\dagger}\right)-A_{h}\left(\mathcal{I}_{h} q^{\dagger}\right)\right] U_{h}^{n}\left(\mathcal{I}_{h} q^{\dagger}\right), \quad n=1,2, \ldots, N,
$$

where $A_{h}\left(q^{\dagger}\right), A_{h}\left(\mathcal{I}_{h} q^{\dagger}\right): X_{h} \rightarrow X_{h}$ are the discrete analogues of the elliptic operators $A\left(q^{\dagger}\right)$ and $A\left(\mathcal{I}_{h} q^{\dagger}\right)$ associated with $q^{\dagger}$ and $\mathcal{I}_{h} q^{\dagger}$, respectively. Thus, it can be written as

$$
\rho_{h}^{n}=\tau \sum_{i=1}^{n} E_{h, \tau}^{n-i}\left[A_{h}\left(q^{\dagger}\right)-A_{h}\left(\mathcal{I}_{h} q^{\dagger}\right)\right] U_{h}^{i}\left(\mathcal{I}_{h} q^{\dagger}\right),
$$

where $E_{h, \tau}^{n}$ is the fully discrete solution operator, which satisfies that for all $v_{h} \in X_{h}$ $[25]$

$$
\begin{aligned}
\left\|E_{h, \tau}^{n} v_{h}\right\|_{L^{2}(\Omega)} & =\left\|A_{h}\left(q^{\dagger}\right)^{\frac{1}{2}} E_{h, \tau}^{n}\left(A_{h}\left(q^{\dagger}\right)^{-\frac{1}{2}} v_{h}\right)\right\|_{L^{2}(\Omega)} \\
& \leq c t_{n+1}^{-1+\frac{\alpha}{2}}\left\|A_{h}\left(q^{\dagger}\right)^{-\frac{1}{2}} v_{h}\right\|_{L^{2}(\Omega)} \leq c t_{n+1}^{-1+\frac{\alpha}{2}}\left\|v_{h}\right\|_{H^{-1}(\Omega)} .
\end{aligned}
$$

It follows from this estimate and the solution representation (4.4) that

$$
\left\|\rho_{h}^{n}\right\|_{L^{2}(\Omega)} \leq c \tau \sum_{i=1}^{n} t_{n}^{-1+\frac{\alpha}{2}}\left\|\left[A_{h}\left(\mathcal{I}_{h} q^{\dagger}\right)-A_{h}\left(q^{\dagger}\right)\right] U_{h}^{n}\left(\mathcal{I}_{h} q^{\dagger}\right)\right\|_{H^{-1}(\Omega)}
$$

Copyright (c) by SIAM. Unauthorized reproduction of this article is prohibited. 
Further, the definitions of $P_{h}$ and $A_{h}$ and the $H^{1}(\Omega)$-stability of $P_{h}$ yield

$$
\begin{aligned}
&\left\|\left[A_{h}\left(\mathcal{I}_{h} q^{\dagger}\right)-A_{h}\left(q^{\dagger}\right)\right] U_{h}^{n}\left(\mathcal{I}_{h} q^{\dagger}\right)\right\|_{H^{-1}(\Omega)} \\
&=\sup _{v \in \dot{H}^{1}} \frac{\left\langle\left[A_{h}\left(\mathcal{I}_{h} q^{\dagger}\right)-A_{h}\left(q^{\dagger}\right)\right] U_{h}^{n}\left(\mathcal{I}_{h} q^{\dagger}\right), v\right\rangle}{\|v\|_{\dot{H}^{1}(\Omega)}} \\
&=\sup _{v \in \dot{H}^{1}} \frac{\left\langle\left(q^{\dagger}-\mathcal{I}_{h} q^{\dagger}\right) \nabla U_{h}^{n}\left(\mathcal{I}_{h} q^{\dagger}\right), \nabla P_{h} v\right\rangle}{\|v\|_{\dot{H}^{1}(\Omega)}} \leq c h^{2}\left\|q^{\dagger}\right\|_{W^{2, \infty}(\Omega)}\left\|\nabla U_{h}^{n}\left(\mathcal{I}_{h} q^{\dagger}\right)\right\|_{L^{2}(\Omega)},
\end{aligned}
$$

since $q \in W^{2, \infty}(\Omega)$ by Assumption 4.1(i) and (3.2). Thus,

$$
\left\|\rho_{h}^{n}\right\|_{L^{2}(\Omega)} \leq c h^{2} \tau \sum_{i=1}^{n} t_{n}^{-1+\frac{\alpha}{2}} \leq c h^{2} \int_{0}^{T} t^{-1+\frac{\alpha}{2}} \mathrm{~d} t \leq c h^{2} .
$$

This and the triangle inequality complete the proof of the lemma.

Next we give an error estimate on the CQ approximation of the fractional derivative. The proof is similar to [27, Lemma 4.2] and is given in Appendix A for completeness.

LEMma 4.3. Let $q^{\dagger}$ be the exact diffusion coefficient and $u \equiv u\left(q^{\dagger}\right)$ be the solution to problem (2.2). Then under Assumption 4.1, there holds

$$
\left\|\bar{\partial}_{\tau}^{\alpha}\left(u\left(t_{n}\right)-u_{0}\right)-\partial_{t}^{\alpha}\left(u\left(t_{n}\right)-u_{0}\right)\right\|_{L^{2}(\Omega)} \leq c \tau t_{n}^{-1} .
$$

The next lemma gives a quadrature error estimate.

Lemma 4.4. Let $q^{\dagger}$ be the exact diffusion coefficient and $u \equiv u\left(q^{\dagger}\right)$ the corresponding solution to problem (2.2). Then under Assumption 4.1,

$$
\sum_{n=0}^{N} a_{i}\left\|u\left(t_{n}\right)-z^{\delta}\left(t_{n}\right)\right\|_{L^{2}(\Omega)}^{2} \leq c\left(\delta^{2}+\tau^{1+\alpha}\right) .
$$

Proof. Let $g(t)=z^{\delta}(t)-u(t)$. By the regularity estimate (4.1) and Assumption 4.1 ,

$$
\|g\|_{C\left([0, T] ; L^{2}(\Omega)\right)} \leq c, \quad\left\|g^{\prime}(t)\right\|_{L^{2}(\Omega)} \leq c t^{\alpha-1}, \quad \text { and } \quad\left\|g^{\prime \prime}(t)\right\|_{L^{2}(\Omega)} \leq c t^{\alpha-2} .
$$

By the triangle inequality, we have

$$
\begin{aligned}
& \left|\tau \sum_{n=0}^{N} a_{i}\left\|g\left(t_{n}\right)\right\|_{L^{2}(\Omega)}^{2}-\sum_{n=1}^{N} \int_{t_{n-1}}^{t_{n}}\|g(t)\|_{L^{2}(\Omega)}^{2} \mathrm{~d} t\right| \\
& \quad \leq \sum_{n=1}^{N}\left|\int_{t_{n-1}}^{t_{n}}\|g(t)\|_{L^{2}(\Omega)}^{2} \mathrm{~d} t-\frac{\tau}{2}\left(\left\|g\left(t_{n-1}\right)\right\|_{L^{2}(\Omega)}^{2}+\left\|g\left(t_{n}\right)\right\|_{L^{2}(\Omega)}^{2}\right)\right|:=\sum_{n=1}^{N} \mathrm{I}_{n} .
\end{aligned}
$$

Next we analyze the two cases $n=1$ and $n>1$ separately. First, for the case $n=1$, $\mathrm{I}_{1} \leq\left|\int_{0}^{\tau}\left(\|g(t)\|_{L^{2}(\Omega)}^{2}-\left\|g\left(t_{0}\right)\right\|_{L^{2}(\Omega)}^{2}\right) \mathrm{d} t\right|+\left|\int_{0}^{\tau}\left(\|g(t)\|_{L^{2}(\Omega)}^{2}-\|g(\tau)\|_{L^{2}(\Omega)}^{2}\right) \mathrm{d} t\right|:=\mathrm{I}_{1,0}+\mathrm{I}_{1,1}$.

Using (4.5), the term $\mathrm{I}_{1,0}$ can be bounded by

$$
\mathrm{I}_{1,0} \leq c\|g(t)\|_{C\left([0, \tau] ; L^{2}(\Omega)\right)} \int_{0}^{\tau}\|g(0)-g(t)\|_{L^{2}(\Omega)} \mathrm{d} t \leq c \tau \int_{0}^{\tau}\left\|g^{\prime}(s)\right\|_{L^{2}(\Omega)} \mathrm{d} s \leq c \tau^{1+\alpha} .
$$

Copyright (c) by SIAM. Unauthorized reproduction of this article is prohibited. 
Similarly, we can deduce $\mathrm{I}_{1,1} \leq c \tau^{1+\alpha}$. Further, for the case $n>1, g(t)$ is smooth, and thus by standard interpolation error estimates, for some $\xi_{n} \in\left[t_{n-1}, t_{n}\right]$, there holds $\mathrm{I}_{n} \leq c \tau^{2} \int_{t_{n-1}}^{t_{n}}\left|\frac{\mathrm{d}^{2}}{\mathrm{~d} t^{2}}\|g(t)\|_{L^{2}(\Omega)}^{2}\right|_{t=\xi_{n}} \mid \mathrm{d} t$. By the bounds in (4.5), $\left|\frac{\mathrm{d}^{2}}{\mathrm{~d} t^{2}}\left\|g\left(\xi_{n}\right)\right\|_{L^{2}(\Omega)}^{2}\right| \leq$ $2\left(\left\|g^{\prime}\left(\xi_{n}\right)\right\|_{L^{2}(\Omega)}^{2}+\left\|g\left(\xi_{n}\right)\right\|_{L^{2}(\Omega)}\left\|g^{\prime \prime}\left(\xi_{n}\right)\right\|_{L^{2}(\Omega)}\right) \leq c t_{n-1}^{\alpha-2}$. The last two estimates together imply

$$
\sum_{n=2}^{N} \mathrm{I}_{n} \leq c \tau^{3} \sum_{n=2}^{N} t_{n-1}^{\alpha-2} \leq c \tau^{1+\alpha} .
$$

Then the assertion follows from the triangle inequality and the definition of the noise level.

Remark 4.5. One can only obtain an $O\left(\tau+\delta^{2}\right)$ rate for the discrete objective function $J_{\gamma, h, \tau}$ in (3.6). Then the $\alpha$ exponent in Lemma 4.4 reflects the limited temporal smoothing property of the solution $u(t)$ : the larger the fractional order $\alpha$ is, the smoother in time the solution $u(t)$ becomes and the faster the quadrature error decays.

The next result gives a priori bounds on $q_{h}^{*}$ and the approximation $U_{h}^{n}\left(q_{h}^{*}\right)$. This result will play a crucial role in the analysis below.

LEMMA 4.6. Let $q^{\dagger}$ be the exact coefficient and $u \equiv u\left(q^{\dagger}\right)$ the solution to problem (2.2). Let $q_{h}^{*} \in \mathcal{A}_{h}$ be the solution to problem (4.2)-(4.3) and $\left\{U_{h}^{n}\left(q_{h}^{*}\right)\right\}_{n=1}^{N}$ the fully discrete solution to problem (3.7). Then under Assumption 4.1, with $\ell_{h}=|\log h|$, there holds

$$
\tau \sum_{n=1}^{N}\left\|U_{h}^{n}\left(q_{h}^{*}\right)-u\left(t_{n}\right)\right\|_{L^{2}(\Omega)}^{2}+\gamma\left\|\nabla q_{h}^{*}\right\|_{L^{2}(\Omega)}^{2} \leq c\left(\tau^{1+\alpha}+h^{4} \ell_{h}^{2}+\delta^{2}+\gamma\right) .
$$

Proof. By the minimizing property of $q_{h}^{*} \in \mathcal{A}_{h}$ and $\mathcal{I}_{h} q^{\dagger} \in \mathcal{A}_{h}$, we deduce $J_{\gamma, h, \tau}\left(q_{h}^{*}\right) \leq J_{\gamma, h, \tau}\left(\mathcal{I}_{h} q^{\dagger}\right)$. By the triangle inequality, we derive

$$
\begin{aligned}
\tau \sum_{n=1}^{N}\left\|U_{h}^{n}\left(q_{h}^{*}\right)-u\left(t_{n}\right)\right\|_{L^{2}(\Omega)}^{2} \leq & c \tau \sum_{n=1}^{N}\left\|U_{h}^{n}\left(q_{h}^{*}\right)-z^{\delta}\left(t_{n}\right)\right\|_{L^{2}(\Omega)}^{2} \\
& +c \tau \sum_{n=0}^{N} a_{n}\left\|z^{\delta}\left(t_{n}\right)-u\left(t_{n}\right)\right\|_{L^{2}(\Omega)}^{2}
\end{aligned}
$$

These two inequalities and Lemma 4.4 imply

$$
\begin{aligned}
& \tau \sum_{n=1}^{N}\left\|U_{h}^{n}\left(q_{h}^{*}\right)-u\left(t_{n}\right)\right\|_{L^{2}(\Omega)}^{2}+\gamma\left\|\nabla q_{h}^{*}\right\|_{L^{2}(\Omega)}^{2} \\
& \quad \leq c \tau \sum_{n=1}^{N}\left\|U_{h}^{n}\left(\mathcal{I}_{h} q^{\dagger}\right)-z^{\delta}\left(t_{n}\right)\right\|_{L^{2}(\Omega)}^{2}+c \gamma\left\|\nabla \mathcal{I}_{h} q^{\dagger}\right\|_{L^{2}(\Omega)}^{2}+c\left(\delta^{2}+\tau^{1+\alpha}\right) .
\end{aligned}
$$

Since $q^{\dagger} \in W^{1, \infty}(\Omega)$ by Assumption 4.1, $\left\|\nabla \mathcal{I}_{h} q^{\dagger}\right\|_{L^{2}(\Omega)} \leq c$ (cf. (3.2)). Further, by Lemma 4.2 , we have

$$
\begin{aligned}
\left\|U_{h}^{n}\left(\mathcal{I}_{h} q^{\dagger}\right)-z^{\delta}\left(t_{n}\right)\right\|_{L^{2}(\Omega)}^{2} & \leq 2\left\|U_{h}^{n}\left(\mathcal{I}_{h} q^{\dagger}\right)-u\left(t_{n}\right)\right\|_{L^{2}(\Omega)}^{2}+2\left\|u\left(t_{n}\right)-z^{\delta}\left(t_{n}\right)\right\|_{L^{2}(\Omega)}^{2} \\
& \leq c\left(\tau t_{n}^{\alpha-1}+h^{2} \ell_{h}\right)^{2}+c\left\|u\left(t_{n}\right)-z^{\delta}\left(t_{n}\right)\right\|_{L^{2}(\Omega)}^{2} .
\end{aligned}
$$

Copyright $\odot$ by SIAM. Unauthorized reproduction of this article is prohibited. 
Consequently,

$$
\begin{aligned}
& \tau \sum_{n=1}^{N}\left\|\nabla\left(U_{h}^{n}\left(\mathcal{I}_{h} q^{\dagger}\right)-z^{\delta}\left(t_{n}\right)\right)\right\|_{L^{2}(\Omega)}^{2} \\
& \quad \leq c \tau \sum_{n=1}^{N}\left(t_{n}^{\alpha-1} \tau+h^{2} \ell_{h}\right)^{2}+c \sum_{n=0}^{N} a_{n}\left\|u\left(t_{n}\right)-z^{\delta}\left(t_{n}\right)\right\|_{L^{2}(\Omega)}^{2} \\
& \quad \leq c \tau^{3} \sum_{n=1}^{N} t_{n}^{\alpha-2}+c h^{4} \ell_{h}^{2}+c\left(\tau^{1+\alpha}+\delta^{2}\right) \\
& \quad \leq c\left(\tau^{1+\alpha}+h^{4} \ell_{h}^{2}+\delta^{2}\right)
\end{aligned}
$$

Combining the preceding estimates completes the proof of the lemma.

We shall also need the following lemma on backward Euler CQ.

Lemma 4.7. Let $q^{\dagger}$ be the exact coefficient and $u \equiv u\left(q^{\dagger}\right)$ the corresponding solution to problem (1.1). Then for $\varphi^{m}=\frac{q^{\dagger}-q_{h}^{*}}{q^{\dagger}} u\left(t_{m}\right)$, and any $\epsilon \in\left(0, \min \left(\frac{1}{2}, 1-\alpha\right)\right)$, there holds

$$
\left\|\tau^{-\alpha} \sum_{n=j}^{m} b_{n-j}^{(\alpha)} P_{h}\left(\varphi^{n}-\varphi^{m}\right)\right\|_{L^{2}(\Omega)} \leq c_{T, \epsilon} t_{j}^{-\epsilon} .
$$

Proof. By the associativity of CQ from (3.5), i.e., $\bar{\partial}_{\tau}^{\alpha} \varphi^{n}=\bar{\partial}_{\tau}^{\alpha-1} \bar{\partial}_{\tau} \varphi^{n}$, if $\varphi^{0}=0$,

$$
\mathrm{I}:=\tau^{-\alpha} \sum_{n=j}^{m} b_{n-j}^{(\alpha)} P_{h}\left(\varphi^{n}-\varphi^{m}\right)=\tau^{1-\alpha} \sum_{n=j}^{m} b_{n-j}^{(\alpha-1)} \frac{P_{h} \varphi^{n}-P_{h} \varphi^{n+1}}{\tau} .
$$

Thus, the $L^{2}(\Omega)$-stability of $P_{h}$, the bound on $\left|b_{j}^{(\alpha-1)}\right| \leq c(j+1)^{-\alpha}$, and (4.1) imply

$$
\begin{aligned}
\|\mathrm{I}\|_{L^{2}(\Omega)} & \leq \tau^{1-\alpha} \sum_{n=j}^{m}\left|b_{n-j}^{(\alpha-1)}\right|\left\|\frac{\varphi^{n}-\varphi^{n+1}}{\tau}\right\|_{L^{2}(\Omega)} \leq c \tau^{1-\alpha} \sum_{n=j}^{m}(n-j+1)^{-\alpha}\left\|\varphi^{\prime}\left(\xi_{n}\right)\right\|_{L^{2}(\Omega)} \\
& \leq c \tau^{1-\alpha} \sum_{n=j}^{m}(n-j+1)^{-\alpha} t_{n}^{\alpha-1} \leq c \int_{t_{j}}^{t_{m}}\left(s-t_{j}+\tau\right)^{-\alpha} s^{\alpha+\epsilon-1} \mathrm{~d} s t_{j}^{-\epsilon}=: g\left(t_{j}\right) t_{j}^{-\epsilon},
\end{aligned}
$$

where $\xi_{n} \in\left[t_{n}, t_{n+1}\right]$. We claim that the integral $g\left(t_{j}\right)$ is decreasing in $t_{j} \in\left[\tau, t_{m}\right]$. Indeed, for any $0<\bar{t}_{1}<\bar{t}_{2} \leq t_{m}$, by changing of variables, there holds

$$
\begin{aligned}
g\left(\bar{t}_{1}\right) & :=\int_{\bar{t}_{1}}^{t_{m}}\left(s-\bar{t}_{1}+\tau\right)^{-\alpha} s^{\alpha+\epsilon-1} \mathrm{~d} s \\
& =\int_{\bar{t}_{1}}^{t_{m}-\left(\bar{t}_{2}-\bar{t}_{1}\right)}\left(s-\bar{t}_{1}+\tau\right)^{-\alpha} s^{\alpha+\epsilon-1} \mathrm{~d} s+\int_{t_{m}-\left(\bar{t}_{2}-\bar{t}_{1}\right)}^{t_{m}}\left(s-\bar{t}_{1}+\tau\right)^{-\alpha} s^{\alpha+\epsilon-1} \mathrm{~d} s \\
& \geq g\left(\bar{t}_{2}\right)+\int_{t_{m}-\left(\bar{t}_{2}-\bar{t}_{1}\right)}^{t_{m}}\left(s-\bar{t}_{1}+\tau\right)^{-\alpha} s^{\alpha+\epsilon-1} \mathrm{~d} s \geq g\left(\bar{t}_{2}\right) .
\end{aligned}
$$

Thus, $\|\mathrm{I}\|_{L^{2}(\Omega)} \leq c t_{j}^{-\epsilon} \int_{\tau}^{t_{m}}(s+\tau)^{-\alpha} s^{\alpha+\epsilon-1} \mathrm{~d} s \leq c_{\epsilon} t_{j}^{-\epsilon}$. This completes the proof of the lemma. 
The next theorem represents the main result of this section, i.e., error estimate of the numerical approximation $q_{h}^{*} \in \mathcal{A}_{h}$ in a weighted $L^{2}(\Omega)$ norm, with the weight $q^{\dagger}\left|\nabla u\left(t_{n}\right)\right|^{2}+\left(f\left(t_{n}\right)-\partial_{t}^{\alpha} u\left(t_{n}\right)\right) u\left(t_{n}\right)$. The proof relies crucially on the choice of the novel test function $\varphi=\frac{q^{\dagger}-q_{h}^{*}}{q^{\dagger}} u$.

THEOREM 4.8. Let $q^{\dagger}$ be the exact diffusion coefficient, $u \equiv u\left(q^{\dagger}\right)$ the solution to problem (2.2), and $q_{h}^{*} \in \mathcal{A}_{h}$ the solution to problem (4.2)-(4.3). Then under Assumption 4.1, for $d=1,2$, with $\ell_{h}=|\log h|$ and $\eta=\tau^{\frac{1}{2}+\frac{\alpha}{2}}+h^{2} \ell_{h}+\delta+\gamma^{\frac{1}{2}}$, there holds

$$
\begin{aligned}
& \tau^{2} \sum_{m=1}^{N} \sum_{n=1}^{m} \int_{\Omega}\left(\frac{q^{\dagger}-q_{h}^{*}}{q^{\dagger}}\right)^{2}\left(q^{\dagger}\left|\nabla u\left(t_{n}\right)\right|^{2}+\left(f\left(t_{n}\right)-\partial_{t}^{\alpha} u\left(t_{n}\right)\right) u\left(t_{n}\right)\right) \mathrm{d} x \\
& \quad \leq c\left(h \gamma^{-1} \eta+h \gamma^{-\frac{1}{2}}+h^{-1} \gamma^{-\frac{1}{2}} \eta\right) \eta .
\end{aligned}
$$

Proof. For any test function $\varphi$ to be specified below, we have the splitting

$$
\begin{aligned}
& \left(\left(q^{\dagger}-q_{h}^{*}\right) \nabla u\left(t_{n}\right), \nabla \varphi\right) \\
& \quad=\left(\left(q^{\dagger}-q_{h}^{*}\right) \nabla u\left(t_{n}\right), \nabla\left(\varphi-P_{h} \varphi\right)\right)+\left(q^{\dagger} \nabla u\left(t_{n}\right)-q_{h}^{*} \nabla u\left(t_{n}\right), \nabla P_{h} \varphi\right) .
\end{aligned}
$$

Thus, applying integration by parts to the first term leads to

$$
\begin{aligned}
\left(\left(q^{\dagger}-q_{h}^{*}\right) \nabla u\left(t_{n}\right), \nabla \varphi\right)= & -\left(\nabla \cdot\left(\left(q^{\dagger}-q_{h}^{*}\right) \nabla u\left(t_{n}\right)\right), \varphi-P_{h} \varphi\right) \\
& +\left(q_{h}^{*} \nabla\left(U_{h}^{n}\left(q_{h}^{*}\right)-u\left(t_{n}\right)\right), \nabla P_{h} \varphi\right) \\
& +\left(q^{\dagger} \nabla u\left(t_{n}\right)-q_{h}^{*} \nabla U_{h}^{n}\left(q_{h}^{*}\right), \nabla P_{h} \varphi\right)=\sum_{i=1}^{3} \mathrm{I}_{i}^{n} .
\end{aligned}
$$

Next we bound the three terms. Direct computation with the triangle inequality gives

$$
\begin{aligned}
\| \nabla & \cdot\left(\left(q^{\dagger}-q_{h}^{*}\right) \nabla u\left(t_{n}\right)\right) \|_{L^{2}(\Omega)} \\
\leq & \left\|\nabla q^{\dagger}\right\|_{L^{\infty}(\Omega)}\left\|\nabla u\left(t_{n}\right)\right\|_{L^{2}(\Omega)}+\left\|q^{\dagger}-q_{h}^{*}\right\|_{L^{\infty}(\Omega)}\left\|\Delta u\left(t_{n}\right)\right\|_{L^{2}(\Omega)} \\
& +\left\|\nabla q_{h}^{*}\right\|_{L^{2}(\Omega)}\left\|\nabla u\left(t_{n}\right)\right\|_{L^{\infty}(\Omega)} .
\end{aligned}
$$

In view of the regularity estimate (4.1), we derive

$$
\begin{aligned}
\left\|\nabla \cdot\left(q^{\dagger}-q_{h}^{*}\right) \nabla u\left(t_{n}\right)\right\|_{L^{2}(\Omega)} & \leq c+\left\|\nabla q_{h}^{*}\right\|_{L^{2}(\Omega)}\left\|\nabla u\left(t_{n}\right)\right\|_{L^{\infty}(\Omega)} \\
& \leq c\left(1+t_{n}^{\min \left(0,1-\frac{d}{2}-\epsilon\right) \frac{\alpha}{2}}\left\|\nabla q_{h}\right\|_{L^{2}(\Omega)}\right),
\end{aligned}
$$

where the second line is due to Sobolev embedding $\|\nabla u\|_{L^{\infty}(\Omega)} \leq c\|u\|_{H^{s}(\Omega)}$ with $s>\frac{d}{2}+1$ (by the convexity of the domain and elliptic regularity [10, Corollary 19.7, p. 166]). This and the Cauchy-Schwarz inequality imply that the first term $I_{1}^{n}$ is bounded by

$$
\left|I_{1}^{n}\right| \leq c\left(1+\left\|\nabla q_{h}\right\|_{L^{2}(\Omega)}\right)\left\|\varphi-P_{h} \varphi\right\|_{L^{2}(\Omega)} .
$$

Now we choose the test function $\varphi$ to be $\varphi \equiv \varphi^{n}=\frac{q^{\dagger}-q_{h}^{*}}{q^{\dagger}} u\left(t_{n}\right) \in H_{0}^{1}(\Omega)$, and then straightforward computation gives $\nabla \varphi^{n}=\left(q^{\dagger-1} \nabla\left(q^{\dagger}-q_{h}^{*}\right)-q^{\dagger-2}\left(q^{\dagger}-q_{h}^{*}\right) \nabla q^{\dagger}\right) u\left(t_{n}\right)+$ 
$q^{\dagger-1}\left(q^{\dagger}-q_{h}^{*}\right) \nabla u\left(t_{n}\right)$. By the box constraint of $\mathcal{A}$ and the regularity estimate (4.1), we have

$\left\|\nabla \varphi^{n}\right\|_{L^{2}(\Omega)} \leq c\left[\left(1+\left\|\nabla q_{h}^{*}\right\|_{L^{2}(\Omega)}\right)\left\|u\left(t_{n}\right)\right\|_{L^{\infty}(\Omega)}+\left\|\nabla u\left(t_{n}\right)\right\|_{L^{2}(\Omega)}\right] \leq c\left(1+\left\|\nabla q_{h}^{*}\right\|_{L^{2}(\Omega)}\right)$,

and the approximation property of the projection operator $P_{h}$ implies $\left\|\varphi^{n}-P_{h} \varphi^{n}\right\|_{L^{2}(\Omega)}$ $\leq \operatorname{ch}\left\|\nabla \varphi^{n}\right\|_{L^{2}(\Omega)} \leq \operatorname{ch}\left(1+\left\|\nabla q_{h}^{*}\right\|_{L^{2}(\Omega)}\right)$. Thus, by Lemma 4.6, the term $\mathrm{I}_{1}^{n}$ is bounded by

$$
\begin{aligned}
\left|I_{1}^{n}\right| & \leq c h t_{n}^{\min \left(0,1-\frac{d}{2}-\epsilon\right) \frac{\alpha}{2}}\left(1+\left\|\nabla q_{h}^{*}\right\|_{L^{2}(\Omega)}\right)^{2} \\
& \leq c t_{n}^{\min \left(0,1-\frac{d}{2}-\epsilon\right) \frac{\alpha}{2}} h\left(1+\gamma^{-1} \eta^{2}\right) \leq c t_{n}^{\min \left(0,1-\frac{d}{2}-\epsilon\right) \frac{\alpha}{2}} h \gamma^{-1} \eta^{2},
\end{aligned}
$$

which together with the trivial inequality $\tau \sum_{n=1}^{N} t_{n}^{\min \left(0,1-\frac{d}{2}-\epsilon\right) \frac{\alpha}{2}} \leq c$ implies

$$
\tau \sum_{n=1}^{N} \mathrm{I}_{1}^{n} \leq c h \gamma^{-1} \eta^{2} .
$$

For the term $\mathrm{I}_{2}^{n}$, by the triangle inequality, inverse inequality, and $H^{1}(\Omega)$ stability of $P_{h}$, we have

$$
\begin{aligned}
\left\|\nabla\left(u\left(t_{n}\right)-U_{h}^{n}\left(q_{h}^{*}\right)\right)\right\|_{L^{2}(\Omega)} & \leq\left\|\nabla\left(u\left(t_{n}\right)-P_{h} u\left(t_{n}\right)\right)\right\|_{L^{2}(\Omega)}+h^{-1}\left\|P_{h} u\left(t_{n}\right)-U_{h}^{n}\left(q_{h}^{*}\right)\right\|_{L^{2}(\Omega)} \\
& \leq c\left(h+h^{-1}\left\|u\left(t_{n}\right)-U_{h}^{n}\left(q_{h}^{*}\right)\right\|_{L^{2}(\Omega)},\right.
\end{aligned}
$$

and consequently, the Cauchy-Schwarz inequality and Lemma 4.6 imply

$$
\begin{aligned}
\tau \sum_{n=1}^{N} \mathrm{I}_{2}^{n} & \leq \tau \sum_{n=1}^{N}\left\|\nabla\left(u\left(t_{n}\right)-U_{h}^{n}\left(q_{h}^{*}\right)\right)\right\|_{L^{2}(\Omega)}\left\|\nabla \varphi^{n}\right\|_{L^{2}(\Omega)} \\
& \leq c\left(h+h^{-1}\left(\tau \sum_{n=1}^{N}\left\|u\left(t_{n}\right)-U_{h}^{n}\left(q_{h}^{*}\right)\right\|_{L^{2}(\Omega)}^{2}\right)^{\frac{1}{2}}\right)\left(1+\left\|\nabla q_{h}^{*}\right\|_{L^{2}(\Omega)}\right) \\
& \leq c\left(h \gamma^{-\frac{1}{2}}+h^{-1} \gamma^{-\frac{1}{2}} \eta\right) \eta
\end{aligned}
$$

Next we bound the third term $\mathrm{I}_{3}^{n}$. It follows directly from (2.2) and (3.7) that

$$
\begin{aligned}
\mathrm{I}_{3}^{n}= & \left(q^{\dagger} \nabla u\left(t_{n}\right)-q_{h}^{*} \nabla U_{h}^{n}\left(q_{h}^{*}\right), \nabla P_{h} \varphi^{n}\right) \\
= & \left(\bar{\partial}_{\tau}^{\alpha}\left(U_{h}^{n}\left(q_{h}^{*}\right)-U_{h}^{0}\right)-\partial_{t}^{\alpha}\left(u\left(t_{n}\right)-u_{0}\right), P_{h} \varphi^{n}\right) \\
= & \left(\bar{\partial}_{\tau}^{\alpha}\left[\left(U_{h}^{n}\left(q_{h}^{*}\right)-U_{h}^{0}\right)-\left(u\left(t_{n}\right)-u_{0}\right)\right], P_{h} \varphi^{n}\right) \\
& +\left(\bar{\partial}_{\tau}^{\alpha}\left(u\left(t_{n}\right)-u_{0}\right)-\partial_{t}^{\alpha}\left(u\left(t_{n}\right)-u_{0}\right), P_{h} \varphi^{n}\right)=: \mathrm{I}_{3,1}^{n}+\mathrm{I}_{3,2}^{n}
\end{aligned}
$$

It remains to bound the two terms $\mathrm{I}_{3,1}^{n}$ and $\mathrm{I}_{3,2}^{n}$ separately. By Lemma 4.3, there holds

$\left|I_{3,2}^{n}\right| \leq\left\|\bar{\partial}_{\tau}^{\alpha}\left(u\left(t_{n}\right)-u_{0}\right)-\partial_{t}^{\alpha}\left(u\left(t_{n}\right)-u_{0}\right)\right\|_{L^{2}(\Omega)}\left\|P_{h} \varphi^{n}\right\|_{L^{2}(\Omega)} \leq c \tau t_{n}^{-1}, \quad n=1,2, \ldots, N$.

Consequently,

$$
\left|\tau^{2} \sum_{m=1}^{N} \sum_{n=1}^{m} I_{3,2}^{n}\right| \leq c \tau^{3} \sum_{m=1}^{N} \sum_{n=1}^{m} t_{n}^{-1} \leq c \tau \log \left(1+t_{N} / \tau\right) .
$$

Copyright $@$ by SIAM. Unauthorized reproduction of this article is prohibited. 
It remains to bound the term $\mathrm{I}_{3,1}^{n}$. Since $U_{h}^{0}\left(q_{h}^{*}\right)=U_{h}^{0}$ and $u(0)=u_{0}$, straightforward computation with summation by parts yields

$$
\begin{aligned}
\tau \sum_{n=1}^{m} \mathrm{I}_{3,1}^{n} & =\tau \sum_{n=0}^{m}\left(\bar{\partial}_{\tau}^{\alpha}\left[\left(U_{h}^{n}\left(q_{h}^{*}\right)-U_{h}^{0}\right)-\left(u\left(t_{n}\right)-u_{0}\right)\right], P_{h} \varphi^{n}\right) \\
& =\tau \sum_{j=0}^{m}\left(\left[\left(U_{h}^{j}\left(q_{h}^{*}\right)-U_{h}^{0}\right)-\left(u\left(t_{j}\right)-u_{0}\right)\right], \tau^{-\alpha} \sum_{n=j}^{m} b_{n-j}^{(\alpha)} P_{h} \varphi^{n}\right) .
\end{aligned}
$$

Next we appeal to the splitting

$\tau^{-\alpha} \sum_{n=j}^{m} b_{n-j}^{(\alpha)} P_{h} \varphi^{n}=\tau^{-\alpha} \sum_{n=j}^{m} b_{n-j}^{(\alpha)} P_{h}\left(\varphi^{n}-\varphi^{m}\right)+\tau^{-\alpha} \sum_{n=j}^{m} b_{n-j}^{(\alpha)} P_{h} \varphi^{m}:=\mathrm{IV}_{j, m}^{1}+\mathrm{IV}_{j, m}^{2}$

By Lemma 3.2, the sum $\mathrm{IV}_{j, m}^{2}$ satisfies

$$
\left\|\mathrm{IV}_{j, m}^{2}\right\|_{L^{2}(\Omega)} \leq c\left\|\varphi^{m}\right\|_{L^{2}(\Omega)}\left(\tau^{-\alpha} \sum_{n=0}^{m-j} b_{n}^{(\alpha)}\right) \leq c t_{m-j+1}^{-\alpha}\left\|\varphi^{m}\right\|_{L^{2}(\Omega)} \leq c t_{m-j+1}^{-\alpha},
$$

since $\left\|\varphi^{m}\right\|_{L^{2}(\Omega)} \leq c$. Then Lemma 4.6 and the Cauchy-Schwarz inequality imply

$$
\begin{gathered}
\tau^{2} \sum_{m=1}^{N} \sum_{j=1}^{m}\left\|U_{h}^{j}\left(q_{h}^{*}\right)-u\left(t_{j}\right)\right\|_{L^{2}(\Omega)}\left\|\mathrm{IV}_{j, m}^{2}\right\|_{L^{2}(\Omega)} \\
\leq c \tau^{2} \sum_{j=1}^{N} \sum_{m=j}^{N}\left\|U_{h}^{j}\left(q_{h}^{*}\right)-u\left(t_{j}\right)\right\|_{L^{2}(\Omega)} t_{m-j+1}^{-\alpha} \\
\leq c\left(\tau \sum_{j=1}^{N}\left\|U_{h}^{j}\left(q_{h}^{*}\right)-u\left(t_{j}\right)\right\|_{L^{2}(\Omega)}^{2}\right)^{\frac{1}{2}} \leq c \eta,
\end{gathered}
$$

where the second inequality is due to $\tau \sum_{m=j}^{N} t_{m-j+1}^{-\alpha} \leq c t_{N-j+1}^{1-\alpha}$. Similarly, by Lemma 4.7,

$$
\begin{gathered}
\tau^{2} \sum_{m=1}^{N} \sum_{j=1}^{m}\left\|U_{h}^{j}\left(q_{h}^{*}\right)-u\left(t_{j}\right)\right\|_{L^{2}(\Omega)}\left\|\mathrm{IV}_{j, m}^{1}\right\|_{L^{2}(\Omega)} \leq c \tau^{2} \sum_{m=1}^{N} \sum_{j=1}^{m}\left\|U_{h}^{j}\left(q_{h}^{*}\right)-u\left(t_{j}\right)\right\|_{L^{2}(\Omega)} t_{j}^{-\epsilon} \\
\quad \leq c \tau \sum_{j=1}^{N}\left\|u_{h}^{j}\left(q_{h}\right)-u\left(t_{j} ; q\right)\right\|_{L^{2}(\Omega)} t_{j}^{-\epsilon} \leq c\left(\tau \sum_{j=1}^{N}\left\|U_{h}^{j}\left(q_{h}^{*}\right)-u\left(t_{j}\right)\right\|_{L^{2}(\Omega)}^{2}\right)^{\frac{1}{2}} \leq c \eta .
\end{gathered}
$$

These two estimates and the triangle inequality lead to

$$
\left|\tau^{2} \sum_{m=1}^{N} \sum_{n=1}^{m}\left(\bar{\partial}_{\tau}^{\alpha}\left[\left(U_{h}^{n}\left(q_{h}^{*}\right)-u_{h}^{0}\right)-\left(u\left(t_{n}\right)-u_{0}\right)\right], P_{h} \varphi^{n}\right)\right| \leq c \eta
$$

The three estimates (4.7), (4.8), and (4.9) together imply

$$
\tau^{2} \sum_{m=1}^{N} \sum_{n=1}^{m}\left(\left(q^{\dagger}-q_{h}^{*}\right) \nabla u\left(t_{n}\right), \nabla \varphi^{n}\right) \leq c\left(h \gamma^{-1} \eta+\gamma^{-\frac{1}{2}} \eta+h^{-1} \gamma^{-\frac{1}{2}} \eta\right) \eta .
$$

Copyright (c) by SIAM. Unauthorized reproduction of this article is prohibited. 
Finally, this and the identity

$$
\left(\left(q^{\dagger}-q_{h}^{*}\right) \nabla u\left(t_{n}\right), \nabla \varphi^{n}\right)=\frac{1}{2} \int_{\Omega}\left(\frac{q^{\dagger}-q_{h}^{*}}{q^{\dagger}}\right)^{2}\left(q^{\dagger}\left|\nabla u\left(t_{n}\right)\right|^{2}+\left(f\left(t_{n}\right)-\partial_{t}^{\alpha} u\left(t_{n}\right)\right) u\left(t_{n}\right)\right) \mathrm{d} x
$$

lead immediately to the desired assertion. This completes the proof of the theorem.

Remark 4.9. The restriction on $d=1,2$ is due to limited regularity pickup on general convex polyhedral domains, in order to ensure $\|\nabla u\|_{L^{\infty}(\Omega)} \leq c\|u\|_{H^{s}(\Omega)} \leq$ c. The result holds also for a polyhedral domain in $\mathbb{R}^{3}$ with suitable conditions $[9$, Theorem 4, p. 18]. One possible strategy to remove the restriction is to use maximal $L^{p}(\Omega)$ regularity [24], instead of the Hilbert space $H^{s}(\Omega)$. Further, it is worth noting that the proof relies heavily on the discrete "integration by parts" formula for convolution quadrature when bounding the term $\mathrm{I}_{3,1}$, which is valid only for the whole interval $[0, T]$ and represents the main obstacle in extending the analysis to the case of partial data, e.g., terminal observation.

The next result is an immediate corollary of Theorem 4.8.

Corollary 4.10. Let $q^{\dagger}$ be the exact diffusion coefficient, $u \equiv u\left(q^{\dagger}\right)$ the solution to problem (2.2), and $q_{h}^{*} \in \mathcal{A}_{h}$ the solution to problem (4.2)-(4.3). Then under Assumption 4.1, for $d=1,2$, there holds (with $\eta=\tau^{\frac{1}{2}+\frac{\alpha}{2}}+h^{2} \ell_{h}+\delta+\gamma^{\frac{1}{2}}$ )

$$
\begin{aligned}
& \int_{0}^{T} \int_{0}^{t} \int_{\Omega}\left(\frac{q^{\dagger}-q_{h}^{*}}{q^{\dagger}}\right)^{2}\left(q^{\dagger}|\nabla u(s)|^{2}+\left(f(s)-\partial_{s}^{\alpha} u(s)\right) u(s)\right) \mathrm{d} x \mathrm{~d} s \mathrm{~d} t \\
& \quad \leq c\left(h \gamma^{-1} \eta+h \gamma^{-\frac{1}{2}}+h^{-1} \gamma^{-\frac{1}{2}} \eta\right) \eta
\end{aligned}
$$

Proof. In view of Theorem 4.8, it suffices to bound the quadrature error:

$$
\begin{aligned}
& \left.\left|\int_{0}^{T} \int_{0}^{t}\right| \nabla u(s)\right|^{2} \mathrm{~d} s \mathrm{~d} t-\tau^{2} \sum_{m=1}^{N} \sum_{n=1}^{m}\left|\nabla u\left(t_{n}\right)\right|^{2} \mid \\
& +\left|\int_{0}^{T} \int_{0}^{t}\left(f(s)-\partial_{s}^{\alpha} u(s)\right) u(s) \mathrm{d} s \mathrm{~d} t-\tau^{2} \sum_{m=1}^{N} \sum_{n=1}^{m}\left(f\left(t_{n}\right)-\partial_{t}^{\alpha} u\left(t_{n}\right)\right) u\left(t_{n}\right)\right|:=\mathrm{I}+\mathrm{II} .
\end{aligned}
$$

It remains to bound the two terms I and II. For the first term,

$$
\begin{aligned}
\mathrm{I} \leq & \left|\sum_{m=1}^{N}\left(\int_{t_{m-1}}^{t_{m}} \int_{0}^{t_{m}}|\nabla u(s)|^{2} \mathrm{~d} s \mathrm{~d} t-\tau^{2} \sum_{n=1}^{m}\left|\nabla u\left(t_{n}\right)\right|^{2}\right)\right| \\
& +\left.\left|\sum_{m=1}^{N} \int_{t_{m-1}}^{t_{m}} \int_{\max \left(t, t_{m-1}\right)}^{t_{m}}\right| \nabla u(s)\right|^{2} \mathrm{~d} s \mathrm{~d} t \mid \\
\leq & \tau \sum_{m=1}^{N} \underbrace{\left.\left|\int_{0}^{t_{m}}\right| \nabla u(s)\right|^{2} \mathrm{~d} s-\tau \sum_{n=1}^{m}\left|\nabla u\left(t_{n}\right)\right|^{2} \mid}_{\mathbf{I}_{m}}+\tau \sum_{m=1}^{N} \int_{t_{m-1}}^{t_{m}}|\nabla u(s)|^{2} \mathrm{~d} s .
\end{aligned}
$$

By the regularity estimate $(4.1),\left\|\nabla u^{\prime}(s)\right\|_{L^{2}(\Omega)} \leq c s^{\frac{\alpha}{2}-1}$ and $\|\nabla u(t)\|_{C\left([0, T] ; L^{2}(\Omega)\right)} \leq$ 
c. Clearly $\tau \sum_{m=1}^{N} \int_{t_{m-1}}^{t_{m}}|\nabla u(s)|^{2} \mathrm{~d} s \leq c \tau$. Further,

$$
\begin{aligned}
\int_{\Omega} \mathrm{I}_{m} \mathrm{~d} x & \leq \sum_{n=1}^{m} \int_{t_{m-1}}^{t_{m}}\left\|\nabla\left(u(s)+u\left(t_{n}\right)\right)\right\|_{L^{2}(\Omega)}\left\|\nabla\left(u(s)-u\left(t_{n}\right)\right)\right\|_{L^{2}(\Omega)} \mathrm{d} s \\
& \leq c\|\nabla u\|_{C\left(\left[0, t_{m}\right] ; L^{2}(\Omega)\right)} \sum_{n=1}^{m} \int_{t_{m-1}}^{t_{m}}\left\|\nabla \int_{s}^{t_{n}} u^{\prime}(\zeta) \mathrm{d} \zeta\right\|_{L^{2}(\Omega)} \mathrm{d} s \\
& \leq c\|\nabla u\|_{C\left(\left[0, t_{m}\right] ; L^{2}(\Omega)\right)} \tau \int_{0}^{t_{m}} s^{\frac{\alpha}{2}-1} \mathrm{~d} s \leq c \tau .
\end{aligned}
$$

The preceding two estimates imply $\int_{\Omega} \mathrm{Id} x \leq c \tau$. The term II can be bounded similarly as $\int_{\Omega} \operatorname{IId} x \leq c \tau|\ln \tau|$. Indeed, under Assumption 4.1(i), the estimate (4.1), and (1.1), we have $\left\|\partial_{t}^{\alpha} u\right\|_{L^{2}(\Omega)} \leq c$ and $\left\|\left(\partial_{t}^{\alpha} u\right)^{\prime}(t)\right\|_{L^{2}(\Omega)} \leq c t^{-1}$, and thus $g(t) \equiv \partial_{t}^{\alpha} u(t)-f(t)$ satisfies $\|g(t)\|_{L^{2}(\Omega)} \leq c$ and $\left\|g^{\prime}(t)\right\|_{L^{2}(\Omega)} \leq c t^{-1}$. Then repeating the argument completes the proof.

Remark 4.11. There has been much interest in deriving error bounds on the Galerkin approximation $q_{h}^{*}$ in the usual $L^{2}(\Omega)$ or Sobolev norm for nonlinear parameter identification problems. However, for the inverse conductivity problem in either the elliptic or parabolic case, such an estimate remains elusive, largely due to a lack of convexity of the regularized problem. The error estimate given in Corollary 4.10 provides one possible route to derive an $L^{2}(\Omega)$ estimate. Indeed, if the exact coefficient $q^{\dagger}$ and the corresponding state $u \equiv u\left(q^{\dagger}\right)$ satisfy

$$
\int_{0}^{T} \int_{0}^{t}\left(q^{\dagger}|\nabla u(s)|^{2}+\left(f(s)-\partial_{s}^{\alpha} u(s)\right) u(s)\right) \mathrm{d} s \mathrm{~d} t>c \quad \text { a.e. } x \in \Omega,
$$

then the usual $L^{2}$ estimate follows directly. In the classical parabolic case, similar structural conditions have been assumed in the literature, e.g., the following characteristic condition [49, 29]: $t^{-1} \int_{0}^{t} \nabla u(x, s) \mathrm{d} s \cdot \nu \geq \delta_{0}>0$ for all $(x, t) \in Q \equiv \Omega \times(0, T]$, where $\nu$ is a constant vector, or [50, Theorem 6.4] $\alpha_{0}\left|\int_{0}^{t} \nabla u(x, s) \mathrm{d} s\right|^{2}+t \int_{0}^{t}\left(u^{\prime}(x, s)-\right.$ $f(x, s)) \mathrm{d} s \geq 0$ a.e. $(x, t) \in Q$. Note that this latter condition is not positively homogeneous (with respect to problem data). Next we comment on the condition (4.10). If $f \equiv 0$ in $Q, u_{0}>0$ in $\Omega$, then the maximum principle for the subdiffusion model [39] implies $u>0$ in $Q$. Further, $w=\partial_{t}^{\alpha} u$ satisfies $\partial_{t}^{\alpha} w-\nabla \cdot\left(q^{\dagger} \nabla w\right)=\partial_{t}^{\alpha} f$ in $Q$, with initial condition $w(0)=\nabla \cdot\left(q^{\dagger} \nabla u_{0}\right)+f(0)$ in $\Omega$ and boundary condition $w=0$ on $\partial \Omega \times(0, T]$. If $\partial_{t}^{\alpha} f(t) \leq 0$ and $\nabla \cdot\left(q^{\dagger} \nabla u_{0}\right)+f(0) \leq 0$, then the maximum principle implies $\partial_{t}^{\alpha} u=w \leq 0$ in $Q$. Further, if $f>0$ in $Q$, then $f-\partial_{t}^{\alpha} u>0$ in $Q$, which implies $\left(f-\partial_{t}^{\alpha} u\right) u>0$ in $Q$. Thus at least a weak version of condition (4.10) holds. We leave further discussions on the condition (4.10) and its analogues to future work.

Remark 4.12. Theorem 4.8 and Corollary 4.10 show that the convergence rate is of order $O\left(\delta^{\frac{1}{4}}\right)$ in the weighted norm, provided that $\gamma=O\left(h^{4}\right)=O\left(\delta^{2}\right)=O\left(\tau^{1+\alpha}\right)$. The error estimate in Theorem 4.8 and Corollary 4.10 is expected to be suboptimal, due to the presence of the factor $h^{-1}$, which arises from the use of inverse inequality in (4.8). It remains unclear how to achieve optimality, even in the standard parabolic case [50].

5. Numerical results and discussions. Now we present numerical results to illustrate the fully discrete scheme (3.6)-(3.7) with one- and two-dimensional examples, with the measurement $z^{\delta}$ over the time interval $\left[T_{0}, T\right]$ (by a straightforward 
TABLE 1

The reconstruction error $\left\|q_{h}^{*}-q^{\dagger}\right\|_{L^{2}(\Omega)}$ for Example 5.1 .

\begin{tabular}{c|cccccc}
\hline$\epsilon$ & 0 & $1.00 \mathrm{e}-3$ & $5.00 \mathrm{e}-3$ & $1.00 \mathrm{e}-2$ & $3.00 \mathrm{e}-2$ & $5.00 \mathrm{e}-2$ \\
$\gamma$ & $1.00 \mathrm{e}-14$ & $1.00 \mathrm{e}-13$ & $3.00 \mathrm{e}-13$ & $5.00 \mathrm{e}-13$ & $1.00 \mathrm{e}-12$ & $3.00 \mathrm{e}-12$ \\
\hline$\alpha=0.25$ & $7.75 \mathrm{e}-3$ & $9.95 \mathrm{e}-3$ & $1.33 \mathrm{e}-2$ & $1.53 \mathrm{e}-2$ & $2.50 \mathrm{e}-2$ & $3.64 \mathrm{e}-2$ \\
$\alpha=0.50$ & $8.73 \mathrm{e}-3$ & $1.00 \mathrm{e}-2$ & $1.33 \mathrm{e}-2$ & $1.50 \mathrm{e}-2$ & $2.65 \mathrm{e}-2$ & $4.11 \mathrm{e}-2$ \\
$\alpha=0.75$ & $9.92 \mathrm{e}-3$ & $1.16 \mathrm{e}-2$ & $1.80 \mathrm{e}-2$ & $2.24 \mathrm{e}-2$ & $3.30 \mathrm{e}-2$ & $5.16 \mathrm{e}-2$ \\
\hline
\end{tabular}

adaptation of the formulation; see Remark 3.8), with $T$ fixed at 1 . Throughout, the corresponding discrete problem is solved by the conjugate gradient (CG) method [3], with the gradient computed using the standard adjoint technique. Unless otherwise stated, the lower and upper bounds in the admissible set $\mathcal{A}$ are taken to be $c_{0}=0.5$ and $c_{1}=5$, respectively, and are enforced by a projection step after each CG iteration. The minimization method converges generally within tens of iterations. The noisy data $z^{\delta}$ is generated by

$$
z^{\delta}(x, t)=u\left(q^{\dagger}\right)(x, t)+\epsilon \sup _{(x, t) \in \Omega \times\left[T_{0}, T\right]}|u(x, t)| \xi(x, t), \quad(x, t) \in \Omega \times\left[T_{0}, T\right],
$$

where $\xi(x, t)$ follows the standard Gaussian distribution, and $\epsilon \geq 0$ denotes the (relative) noise level. The noisy data $z^{\delta}$ is first generated on a fine spatial-temporal mesh and then interpolated to a coarse spatial/temporal mesh for the inversion step. The scalar $\gamma$ in the functional $J_{\gamma}$ plays an important role in determining the accuracy of the reconstructions, but it is notoriously challenging to choose (see, e.g., [20]). In our experiments, its value is determined by a trial and error manner, first for the fractional order $\alpha=0.50$ and then for the cases $\alpha=0.25$ and $\alpha=0.75$, which might be suboptimal but works reasonably well in practice.

5.1. Numerical results in one spatial dimension. First we present numerical results for two examples on unit interval $\Omega=(0,1)$. The reference data $u\left(q^{\dagger}\right)$ is computed with a mesh size $h=1 / 400$ and time step size $\tau=1 / 2048$, and the inversion step is carried out with a mesh size $h=1 / 200$ and time step size $\tau=1 / 1024$, unless otherwise specified.

The first example has a smooth exact coefficient $q^{\dagger}$, and the problem is homogeneous.

Example 5.1. $u_{0}=x(1-x), f \equiv 0, q^{\dagger}=2+\sin (2 \pi x)$.

First, we let $T_{0}=0.75$ and study how the reconstruction error changes with respect to different parameters. The numerical results for the example with different noise levels $\epsilon$, and fixed $h$ and $\tau$, are summarized in Table 1 . The chosen $\gamma$ is relatively small, since the magnitude of the exact data $u\left(q^{\dagger}\right)$ is actually very small: for example, upon convergence, the functional value $J_{\gamma, h, \tau}\left(q_{h}^{*}\right)$ is about $O\left(10^{-12}\right)$ for exact data and about $O\left(10^{-9}\right)$ for $\epsilon=1.00 \mathrm{e}-2$. Clearly, the $L^{2}(\Omega)$ error $e_{q}$ of the reconstruction $q_{h}^{*}$, i.e., $e_{q}=\left\|q^{\dagger}-q_{h}^{*}\right\|_{L^{2}(\Omega)}$, decreases steadily as the noise level $\epsilon$ tends to zero. (Note that even at $\epsilon=0$, the reconstruction error $e_{q}$ is nonzero due to the presence of discretization errors.) The convergence is consistently observed for all three fractional orders. Interestingly, for a fixed noise level $\epsilon$, as the fractional order $\alpha$ increases from 0.25 to 0.75 , the reconstruction error tends to deteriorate slightly. It might be related to the fact that for homogeneous subdiffusion, the smaller $\alpha$ is, the quicker the state $u(t)$ approaches a "quasi"-steady state; then the inverse problem reduces to the elliptic counterpart, i.e., $-\nabla \cdot(q \nabla u)=f$, which is known to be beneficial 

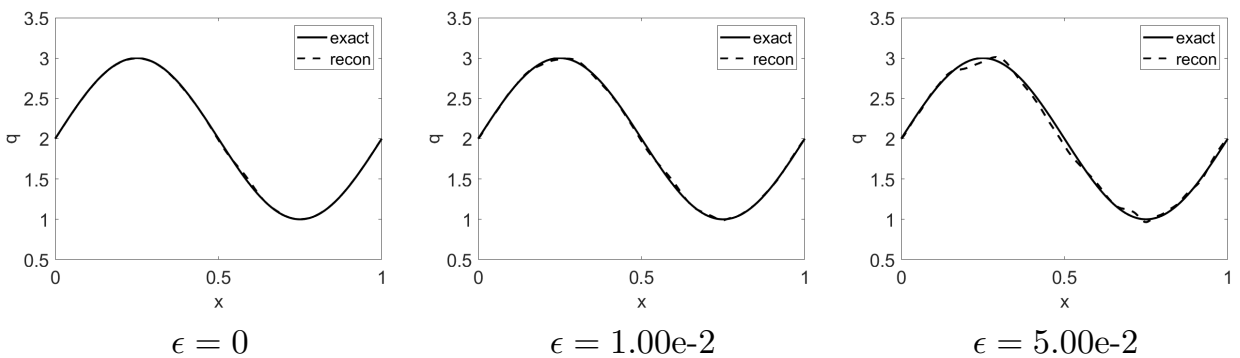

FIG. 1. Numerical reconstructions for Example 5.1 with $\alpha=0.5$.

TABLE 2

Reconstruction errors $\left\|q_{h}^{*}-q^{*}\right\|_{L^{2}(\Omega)}$ for Example 5.1 with $\epsilon=1.00 \mathrm{e}-2$ (and $\left.\beta=5.00 \mathrm{e}-13\right) R$, vs. the mesh size $h=1 / M$ with $\tau$ fixed at $\tau=2^{-10}$.

\begin{tabular}{c|cccccc}
\hline$M$ & 10 & 20 & 40 & 80 & 160 & 320 \\
\hline$\alpha=0.25$ & $5.39 \mathrm{e}-2$ & $2.74 \mathrm{e}-2$ & $2.33 \mathrm{e}-2$ & $1.46 \mathrm{e}-2$ & $2.04 \mathrm{e}-2$ & $1.15 \mathrm{e}-2$ \\
$\alpha=0.50$ & $5.38 \mathrm{e}-2$ & $2.56 \mathrm{e}-2$ & $2.51 \mathrm{e}-2$ & $1.56 \mathrm{e}-2$ & $1.16 \mathrm{e}-2$ & $6.51 \mathrm{e}-3$ \\
$\alpha=0.75$ & $4.61 \mathrm{e}-2$ & $2.57 \mathrm{e}-2$ & $2.26 \mathrm{e}-2$ & $2.41 \mathrm{e}-2$ & $1.14 \mathrm{e}-2$ & $8.00 \mathrm{e}-3$ \\
\hline
\end{tabular}

TABLE 3

Reconstruction errors $\left\|q_{h}^{*}-q^{*}\right\|_{L^{2}(\Omega)}$ for Example 5.1 with $\epsilon=1.00 \mathrm{e}-2$ (and $\beta=5.00 \mathrm{e}-13$ ), vs. the time step size $\tau$, with $h$ fixed at $h=5 \mathrm{e}-3$.

\begin{tabular}{c|cccccc}
\hline$\tau$ & $2^{-5}$ & $2^{-6}$ & $2^{-7}$ & $2^{-8}$ & $2^{-9}$ & $2^{-10}$ \\
\hline$\alpha=0.25$ & $3.78 \mathrm{e}-2$ & $3.88 \mathrm{e}-2$ & $2.03 \mathrm{e}-2$ & $8.30 \mathrm{e}-3$ & $2.38 \mathrm{e}-2$ & $6.27 \mathrm{e}-3$ \\
$\alpha=0.50$ & $3.90 \mathrm{e}-2$ & $3.80 \mathrm{e}-2$ & $1.98 \mathrm{e}-2$ & $1.92 \mathrm{e}-2$ & $2.07 \mathrm{e}-2$ & $8.46 \mathrm{e}-3$ \\
$\alpha=0.75$ & $9.31 \mathrm{e}-2$ & $4.47 \mathrm{e}-2$ & $2.64 \mathrm{e}-2$ & $1.06 \mathrm{e}-2$ & $1.45 \mathrm{e}-2$ & $6.64 \mathrm{e}-3$ \\
\hline
\end{tabular}

for numerical reconstruction [26]. However, the precise mechanism remains to be ascertained. We refer to Figure 1 for exemplary reconstructions: the recoveries are qualitatively comparable with each other and all reasonably accurate for $\epsilon$ up to $\epsilon=5.00 \mathrm{e}-2$. These observations concur well with the numbers in Table 1 .

Next we examine the convergence with respect to the mesh size $h$ and time step size $\tau$; see Tables 2 and 3 for the empirical convergence with respect to $h$ and $\tau$, respectively. The reference regularized solution $q^{*}$ is computed with $h=1 / 800$ and $\tau=1 / 2048$, and it differs slightly from the exact diffusion coefficient $q^{\dagger}$, due to the presence of data noise $(\epsilon=1 \mathrm{e}-2)$. Clearly, the $L^{2}(\Omega)$ error $\left\|q^{*}-q_{h}^{*}\right\|_{L^{2}(\Omega)}$ of the reconstruction $q_{h}^{*}$ (which depends also implicitly on $\tau$ via the optimization problem (3.6)-(3.7)) decreases as either the mesh size $h$ or time step size $\tau$ tends to zero, and the convergence is generally steady. These observations partially confirm the convergence result in Theorem 3.7.

Last, we take $T_{0}=0$ and examine the convergence of the errors $e_{q}=\left\|q^{\dagger}-q_{h}^{*}\right\|_{L^{2}(\Omega)}$ and $e_{u}=\left(\tau \sum_{n=1}^{N}\left\|u\left(t_{n}\right)-U_{h}^{n}\left(q_{h}^{*}\right)\right\|_{L^{2}(\Omega)}^{2}\right)^{\frac{1}{2}}$, with respect to $\epsilon$. Motivated by the error estimates in Theorem 4.8 and Remark 4.12, we fix a small $\tau=1 / 2048$ and let $h=\sqrt{\epsilon}$ and $\gamma=10^{-4} \times \epsilon^{2}$. The errors $e_{q}$ and $e_{u}$ are plotted in Figure 2: a first-order convergence $O(\epsilon)$ is clearly observed. This shows the suboptimality of the theoretical convergence rate in Theorem 4.8. This remains an outstanding question for the analysis of the discrete problem and seems open even for the standard parabolic case.

The second example has a nonsmooth exact coefficient $q^{\dagger}$, and the problem is inhomogeneous. The notation min denotes the pointwise minimum. 

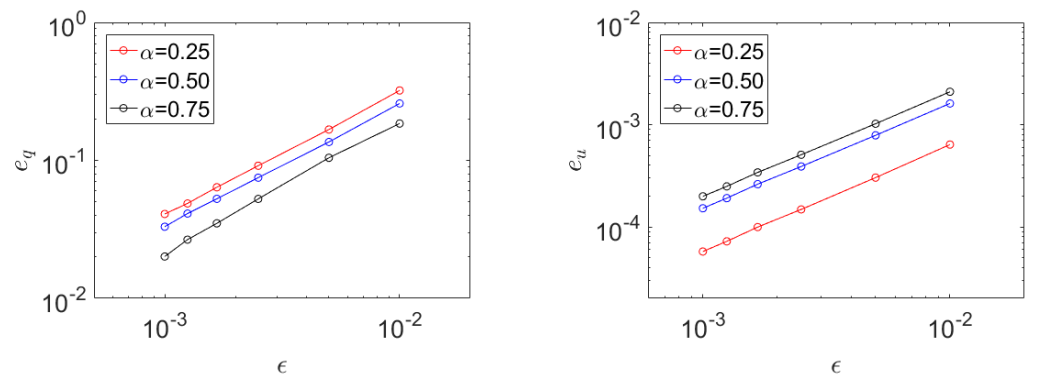

Fig. 2. Plot of $e_{u}$ and $e_{q}$ versus $\epsilon$ with $h=\sqrt{\epsilon}, \gamma=10^{-4} \times \epsilon^{2}$, and $\tau=1 / 2048$.

TABLE 4

Reconstruction error $\left\|q_{h}^{*}-q^{\dagger}\right\|_{L^{2}(\Omega)}$ for Example 5.2.

\begin{tabular}{c|cccccc}
\hline$\epsilon$ & 0 & $1.00 \mathrm{e}-3$ & $5.00 \mathrm{e}-3$ & $1.00 \mathrm{e}-2$ & $3.00 \mathrm{e}-2$ & $5.00 \mathrm{e}-2$ \\
$\gamma$ & $1.00 \mathrm{e}-15$ & $2.00 \mathrm{e}-13$ & $4.00 \mathrm{e}-13$ & $1.00 \mathrm{e}-12$ & $4.00 \mathrm{e}-12$ & $9.00 \mathrm{e}-12$ \\
\hline$\alpha=0.25$ & $4.36 \mathrm{e}-3$ & $7.91 \mathrm{e}-3$ & $1.28 \mathrm{e}-2$ & $1.56 \mathrm{e}-2$ & $2.21 \mathrm{e}-2$ & $3.02 \mathrm{e}-2$ \\
$\alpha=0.50$ & $6.13 \mathrm{e}-3$ & $6.95 \mathrm{e}-3$ & $1.30 \mathrm{e}-2$ & $1.58 \mathrm{e}-2$ & $2.34 \mathrm{e}-2$ & $2.89 \mathrm{e}-2$ \\
$\alpha=0.75$ & $1.04 \mathrm{e}-2$ & $1.14 \mathrm{e}-2$ & $1.44 \mathrm{e}-2$ & $1.54 \mathrm{e}-2$ & $2.18 \mathrm{e}-2$ & $3.23 \mathrm{e}-2$ \\
\hline
\end{tabular}
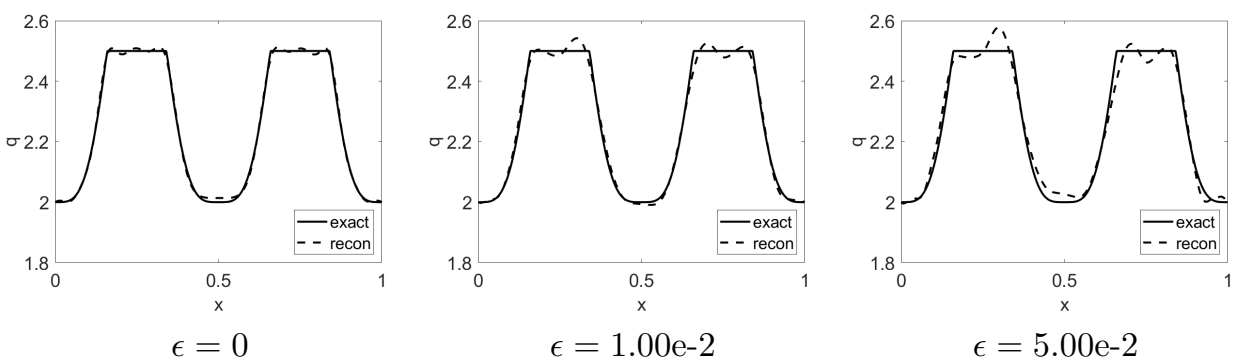

FIG. 3. Numerical reconstructions for Example 5.2 with $\alpha=0.5$.

Example 5.2. $u_{0}(x)=x^{2}(1-x)^{2}, f(x, t)=e^{x(1-x)} x(1-x) t, q^{\dagger}=2+\min \left(\frac{1}{2}, \sin ^{4}\right.$ $(2 \pi x))$, and $T_{0}=0.75$.

The numerical results for the example with different noise levels are given in Table 4 and Figure 3, where the lower and upper bounds in the admissible set $\mathcal{A}$ are taken to be $c_{0}=1.9$ and $c_{1}=2.7$. With this choice, the box constraint becomes active at some CG iterations. The observations from Example 5.1 remain largely valid: the error $e_{q}=\left\|q^{\dagger}-q_{h}^{*}\right\|_{L^{2}(\Omega)}$ decreases as the noise level $\epsilon$ decreases to zero. The results are mostly comparable for all three fractional orders. For high noise levels, e.g., $\epsilon=5.00 \mathrm{e}-2$, the reconstruction error is clearly dominated by the oscillations within the flat regions, which is reminiscent of the Gibbs phenomenon arising from the approximation of the kinks, and also the deviations in the valley. Nonetheless, all the results are fair and represent acceptable approximations.

5.2. Numerical results in two spatial dimensions. Now we present numerical results for the following example on the unit square $\Omega=(0,1)^{2}$. The domain $\Omega$ is first uniformly divided into $M^{2}$ small squares, each with side length $1 / M$, and then a uniform triangulation is obtained by connecting the lower-left and upper-right 
TABLE 5

Reconstruction error $\left\|q_{h}^{*}-q^{\dagger}\right\|_{L^{2}(\Omega)}$ for Example 5.3 .

\begin{tabular}{c|cccccc}
\hline$\epsilon$ & 0 & $1.00 \mathrm{e}-3$ & $5 \mathrm{e}-3$ & $1.00 \mathrm{e}-2$ & $3.00 \mathrm{e}-2$ & $5.00 \mathrm{e}-2$ \\
$\gamma$ & $1.00 \mathrm{e}-14$ & $3.00 \mathrm{e}-12$ & $1.00 \mathrm{e}-11$ & $3.00 \mathrm{e}-11$ & $2.00 \mathrm{e}-10$ & $5.00 \mathrm{e}-10$ \\
\hline$\alpha=0.25$ & $1.51 \mathrm{e}-3$ & $1.75 \mathrm{e}-3$ & $2.87 \mathrm{e}-3$ & $3.64 \mathrm{e}-3$ & $5.82 \mathrm{e}-3$ & $7.81 \mathrm{e}-3$ \\
$\alpha=0.50$ & $1.61 \mathrm{e}-3$ & $1.86 \mathrm{e}-3$ & $2.80 \mathrm{e}-3$ & $3.62 \mathrm{e}-3$ & $6.58 \mathrm{e}-3$ & $9.57 \mathrm{e}-3$ \\
$\alpha=0.75$ & $1.59 \mathrm{e}-3$ & $2.21 \mathrm{e}-3$ & $3.38 \mathrm{e}-3$ & $4.66 \mathrm{e}-3$ & $1.13 \mathrm{e}-2$ & $1.64 \mathrm{e}-2$ \\
\hline
\end{tabular}
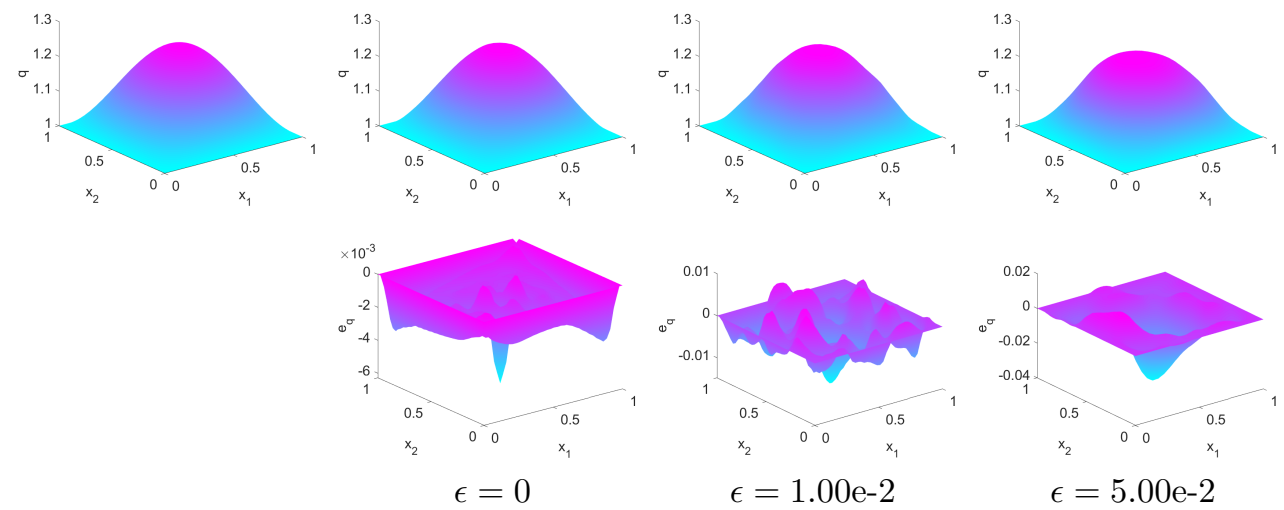

FIG. 4. Numerical reconstructions for Example 5.3 with $\alpha=0.50$.

vertices of each small square. The reference data is first computed on a finer mesh with $M=100$ and a time step size $\tau=1 / 2000$. The inversion is carried out with a mesh $M=40$ and $\tau=1 / 500$.

Example 5.3. $u_{0}\left(x_{1}, x_{2}\right)=x_{1}\left(1-x_{1}\right) \sin \left(\pi x_{2}\right), f \equiv 0, q^{\dagger}\left(x_{1}, x_{2}\right)=1+\sin \left(\pi x_{1}\right) x_{2}$ $\left(1-x_{2}\right)$, and $T_{0}=0.8$.

The numerical results for the example with different noise levels are presented in Table 5 and Figure 4. The empirical observations are in excellent agreement with Example 5.1, e.g., convergence as the noise level $\epsilon$ decreases to zero and slightly improved reconstructions for increasing fractional orders $\alpha$. Figure 4 indicates that the pointwise error $e_{q}=q_{h}^{*}-q^{\dagger}$ lies mainly in recovering the peak, but the overall shape is well recovered.

6. Conclusions. In this work, we have studied the numerical recovery of a spatially dependent diffusion coefficient from the full space-time datum using a regularized least-squares formulation. First, we proved the well-posedness of the continuous formulation, e.g., stability and convergence. Second, we described a fully discrete scheme based on the Galerkin finite element method in space and convolution quadrature in time and showed the convergence of the numerical approximation. Third, we derived error estimates for the numerical approximation under certain regularity conditions on the exact diffusion coefficient and problem data.

This work presents only a first step toward rigorous numerical analysis of the inverse conductivity problem. There are several avenues deserving further research. First, it is important to analyze the formally determined case, e.g., terminal data or lateral Cauchy data. This is apparently very challenging, since even for the classical parabolic counterparts, a rigorous error estimate (in either a weighted norm or 
the usual $L^{2}(\Omega)$ ) remains elusive. The techniques in this work also do not extend directly, due to their heavy use of the discrete "integration by parts" formula over the interval $[0, T]$. Second, even for full data, the obtained error estimates remain suboptimal in terms of their dependence with the mesh size $h$, when compared with the empirical convergence rate. Partly, this arises from the inverse inequality, and it remains unclear how to achieve optimality. Third, it is of great interest to recover the fractional order $\alpha$ and the diffusion coefficient $q$ simultaneously, or a space-time dependent diffusion coefficient. Fourth and last, it is of much interest to derive the necessary and sufficient optimality conditions for the regularized formulation, to carry out convergence and error analysis with respect to stationary points, and to develop more efficient numerical algorithms. The optimality system may be derived using the spike variation technique in a fairly general setting (see, e.g., [40] for the standard parabolic case).

\section{Appendix A. Proof of Lemma 4.3.}

The proof relies on the discrete Laplace transform and the following two estimates:

$$
\begin{gathered}
c_{1}|z| \leq\left|\delta_{\tau}\left(e^{-z \tau}\right)\right| \leq c_{2}|z| \quad \forall z \in \Gamma_{\theta, \delta}^{\tau}, \\
\left|\delta_{\tau}\left(e^{-z \tau}\right)\right| \leq|z| \sum_{k=1}^{\infty} \frac{|z \tau|^{k-1}}{k !} \leq|z| e^{|z| \tau} \quad \forall z \in \Sigma_{\theta}
\end{gathered}
$$

with $\Sigma_{\theta}=\{z \in \mathbb{C}: z \neq 0,|\arg (z)| \leq \theta\}$ and $\Gamma_{\theta, \delta}^{\tau}=\left\{z=r e^{ \pm \mathrm{i} \theta}, \delta \leq r \leq \frac{\pi \sin \theta}{\tau}\right\} \cup\{z=$ $\left.\delta e^{\mathrm{i} \varphi}:|\varphi| \leq \theta\right\}$, where $\theta \in\left(\frac{\pi}{2}, \pi\right)$ is fixed, and the resolvent estimate

$$
\left\|(z-A(q))^{-1}\right\| \leq c|z|^{-1} \quad \forall z \in \Sigma_{\theta} .
$$

Now let $y(t)=u(t)-u_{0}$. Then $y(t)$ satisfies

$$
\partial_{t}^{\alpha} y(t)-A y(t)+A u_{0}=f(t), \quad 0<t \leq T .
$$

Taking the Laplace transform gives

$$
z^{\alpha} \widehat{y}(z)-A \widehat{y}(z)+z^{-1} A u_{0}=\widehat{f}(z),
$$

i.e., $\widehat{y}(z)=\left(z^{\alpha}-A\right)^{-1}\left(\widehat{f}(z)-z^{-1} A u_{0}\right)$. Since $\widehat{\partial_{t}^{\alpha} y(t)}=z^{\alpha} \widehat{y}(z)$ and $\widehat{\bar{\partial}_{\tau}^{\alpha} y}=\delta_{\tau}(z)^{\alpha} \widehat{y}(z)$, then $w^{n}=\partial_{t}^{\alpha} y\left(t_{n}\right)-\bar{\partial}_{\tau}^{\alpha} y\left(t_{n}\right)$ is represented by

$w^{n}=\frac{1}{2 \pi \mathrm{i}} \int_{\Gamma_{\theta, \delta}^{\tau}} e^{z t_{n}} K(z)\left(z^{-1} A u_{0}-\widehat{f}(z)\right) \mathrm{d} z+\frac{1}{2 \pi \mathrm{i}} \int_{\Gamma_{\theta, \delta} \backslash \Gamma_{\theta, \delta}^{\tau}} e^{z t_{n}} K(z)\left(z^{-1} A u_{0}-\widehat{f}(z)\right) \mathrm{d} z$

with $K(z)=\left(\delta_{\tau}\left(e^{-z \tau}\right)^{\alpha}-z^{\alpha}\right)\left(z^{\alpha}-A\right)^{-1}$. Recall the following estimate:

$$
\left|\delta_{\tau}\left(e^{-z \tau}\right)^{\alpha}-z^{\alpha}\right| \leq c \tau z^{1+\alpha} \quad \forall z \in \Gamma_{\theta, \delta}^{\tau} .
$$

By choosing $\delta=c / t_{n}$ and (A.3), $\mathrm{I}=\frac{1}{2 \pi \mathrm{i}} \int_{\Gamma_{\theta, \delta}^{\tau}} e^{z t_{n}} K(z) z^{-1}\left(A u_{0}-f(0)\right) \mathrm{d} z$ is bounded by

$$
\begin{aligned}
\|\mathrm{I}\|_{L^{2}(\Omega)} & \leq c \tau\left\|A u_{0}-f(0)\right\|_{L^{2}(\Omega)}\left(\int_{\frac{c}{t_{n}}}^{\frac{\pi \sin \theta}{\tau}} e^{-c \rho t_{n}} \mathrm{~d} \rho+\int_{-\theta}^{\theta} c t_{n}^{-1} \mathrm{~d} \theta\right) \\
& \leq c \tau t_{n}^{-1}\left\|A u_{0}-f(0)\right\|_{L^{2}(\Omega)} .
\end{aligned}
$$

Copyright $\odot$ by SIAM. Unauthorized reproduction of this article is prohibited. 
Further, by (A.2), for any $z=\rho e^{ \pm \mathrm{i} \theta} \in \Gamma_{\theta, \delta} \backslash \Gamma_{\theta, \delta}^{\tau}$ and choosing $\theta \in(\pi / 2, \pi)$ close to $\pi$,

$$
\left|e^{z t_{n}}\left(\delta_{\tau}\left(e^{-z \tau}\right)^{\alpha}-z^{\alpha}\right) z^{-1}\right| \leq e^{t_{n} \rho \cos \theta}\left(c|z|^{\alpha} e^{\alpha \rho \tau}+|z|^{\alpha}\right)|z|^{-1} \leq c|z|^{\alpha-1} e^{-c \rho t_{n}} .
$$

Then the term II $=\frac{1}{2 \pi \mathrm{i}} \int_{\Gamma_{\theta, \delta} \backslash \Gamma_{\theta, \delta}^{\tau}} e^{z t_{n}} K(z) z^{-1}\left(A u_{0}-f(0)\right) \mathrm{d} z$ is bounded by

$$
\|\mathrm{II}\|_{L^{2}(\Omega)} \leq c\left\|A u_{0}-f(0)\right\|_{L^{2}(\Omega)} \int_{\frac{\pi \sin \theta}{\tau}}^{\infty} e^{-c \rho t_{n}} \rho^{-1} \mathrm{~d} \rho \leq c \tau t_{n}^{-1}\left\|A u_{0}-f(0)\right\|_{L^{2}(\Omega)} .
$$

In view of the splitting $f(t)=f(0)+t f^{\prime}(0)+{ }_{0} I_{t}^{2} f^{\prime \prime}(t)$, it remains to bound the other two terms. Upon extending $f^{\prime \prime}(t)$ by zero to $\mathbb{R}_{-}$, straightforward computation gives

$$
\begin{aligned}
w^{n}= & -\frac{1}{2 \pi \mathrm{i}} \int_{\Gamma_{\theta, \delta}} e^{z t_{n}} K(z) z^{-2} \mathrm{~d} z f^{\prime}(0) \mathrm{d} s \\
& -\frac{1}{2 \pi \mathrm{i}} \int_{0}^{t_{n}} \int_{\Gamma_{\theta, \delta} \backslash \Gamma_{\theta, \delta}^{\tau}} e^{z\left(t_{n}-s\right)} z^{-2} K(z) \mathrm{d} z f^{\prime \prime}(s) \mathrm{d} s .
\end{aligned}
$$

Then repeating the preceding argument leads to

$$
\left\|w^{n}\right\|_{L^{2}(\Omega)} \leq c \tau\left(\left\|f^{\prime}(0)\right\|_{L^{2}(\Omega)}+\int_{0}^{t_{n}}\left\|f^{\prime \prime}(s)\right\|_{L^{2}(\Omega)} \mathrm{d} s\right) .
$$

Combining the preceding estimates shows the desired assertion.

Acknowledgments. The authors are grateful to two anonymous referees and the editor, Professor Karl Kunisch, for several constructive comments that have led to an improvement in the presentation of the paper.

\section{REFERENCES}

[1] E. E. Adams And L. W. Gelhar, Field study of dispersion in a heterogeneous aquifer: 2. Spatial moments analysis, Water Res. Research, 28 (1992), pp. 3293-3307.

[2] R. A. Adams and J. J. F. Fournier, Sobolev Spaces, 2nd ed., Elsevier/Academic Press, Amsterdam, 2003.

[3] O. M. Alifanov, E. A. Artyukhin, and S. V. Rumyantsev, Extreme Methods for Solving Ill-Posed Problems with Applications to Inverse Heat Transfer Problems, Begell House, New York, 1995.

[4] A. A. Alikhanov, A priori estimates for solutions of boundary value problems for equations of fractional order, Differ. Uravn., 46 (2010), pp. 658-664, https://doi.org/10.1134/ S0012266110050058.

[5] B. Berkowitz, A. Cortis, M. Dentz, And H. Scher, Modeling non-Fickian transport in geological formations as a continuous time random walk, Rev. Geophys., 44 (2006), pp. RG2003, 51 pp.

[6] J.-P. Bouchaud and A. Georges, Anomalous diffusion in disordered media: Statistical mechanisms, models and physical applications, Phys. Rep., 195 (1990), pp. 127-293.

[7] E. Casas, P. I. Kogut, and G. Leugering, Approximation of optimal control problems in the coefficient for the p-Laplace equation. I. Convergence result, SIAM J. Control Optim., 54 (2016), pp. 1406-1422, https://doi.org/10.1137/15M1028108.

[8] J. Cheng, J. Nakagawa, M. Yamamoto, and T. Yamazaki, Uniqueness in an inverse problem for a one-dimensional fractional diffusion equation, Inverse Problems, 25 (2009), 115002, https://doi.org/10.1088/0266-5611/25/11/115002.

[9] M. DAuge, Regularity and Singularities in Polyhedral Domains, https://perso.univrennes1.fr/monique.dauge/publis/Talk_Karlsruhe08.pdf, 2019 (last accessed on August 1, 2019).

Copyright $@$ by SIAM. Unauthorized reproduction of this article is prohibited. 
[10] M. Dauge, Elliptic Boundary Value Problems on Corner Domains, Springer, New York, 1988, https://doi.org/10.1007/BFb0086682.

[11] M. Dentz, A. Cortis, H. Scher, and B. Berkowitz, Time behavior of solute transport in heterogeneous media: Transition from anomalous to normal transport, Adv. Water Res., 27 (2004), pp. 155-173.

[12] P. DuChateau, R. Thelwell, and G. Butters, Analysis of an adjoint problem approach to the identification of an unknown diffusion coefficient, Inverse Problems, 20 (2004), pp. 601-625, https://doi.org/10.1088/0266-5611/20/2/019.

[13] H. W. Engl, M. Hanke, and A. Neubauer, Regularization of Inverse Problems, Kluwer Academic, Dordrecht, 1996.

[14] H. W. Engl And J. ZOU, A new approach to convergence rate analysis of Tikhonov regularization for parameter identification in heat conduction, Inverse Problems, 16 (2000), pp. 1907-1923, https://doi.org/10.1088/0266-5611/16/6/319.

[15] A. Ern and J.-L. Guermond, Theory and Practice of Finite Elements, Springer, New York, 2004.

[16] L. C. Evans And R. F. Gariepy, Measure Theory and Fine Properties of Functions, CRC Press, Boca Raton, FL, 2015.

[17] S. Gutman, Identification of discontinuous parameters in flow equations, SIAM J. Control Optim., 28 (1990), pp. 1049-1060, https://doi.org/10.1137/0328057.

[18] Y. Hatano and N. Hatano, Dispersive transport of ions in column experiments: An explanation of long-tailed profiles, Water Res. Research, 34 (1998), pp. 1027-1033.

[19] V. Isakov, Inverse Problems for Partial Differential Equations, 2nd ed., Springer, New York, 2006.

[20] K. Iто And B. Jin, Inverse Problems: Tikhonov Theory and Algorithms, World Scientific, Hackensack, NJ, 2015.

[21] D. JiAnG, Z. Li, Y. LiU, AND M. YAмAмото, Weak unique continuation property and a related inverse source problem for time-fractional diffusion-advection equations, Inverse Problems, 33 (2017), 055013, https://doi.org/10.1088/1361-6420/aa58d1.

[22] B. Jin, R. LAZArov, And Z. Zhou, Two fully discrete schemes for fractional diffusion and diffusion-wave equations with nonsmooth data, SIAM J. Sci. Comput., 38 (2016), pp. A146A170, https://doi.org/10.1137/140979563.

[23] B. Jin, R. LAZAROv, AND Z. ZHOU, Numerical methods for time-fractional evolution equations with nonsmooth data: A concise overview, Comput. Methods Appl. Mech. Engrg., 346 (2019), pp. 332-358, https://doi.org/10.1016/j.cma.2018.12.011.

[24] B. Jin, B. LI, AND Z. ZHou, Discrete maximal regularity of time-stepping schemes for fractional evolution equations, Numer. Math., 138 (2018), pp. 101-131, https://doi.org/10. 1007/s00211-017-0904-8.

[25] B. Jin, B. LI, AND Z. ZHOU, Numerical analysis of nonlinear subdiffusion equations, SIAM J. Numer. Anal., 56 (2018), pp. 1-23, https://doi.org/10.1137/16M1089320.

[26] B. Jin And W. Rundell, A tutorial on inverse problems for anomalous diffusion processes, Inverse Problems, 31 (2015), 035003, https://doi.org/10.1088/0266-5611/31/3/035003.

[27] B. Jin AND Z. ZHOU, Incomplete iterative solution of subdiffusion, Numer. Math., 145 (2020), pp. 693-725, https://doi.org/10.1007/s00211-020-01128-w.

[28] B. Kaltenbacher AND W. Rundell, On an inverse potential problem for a fractional reactiondiffusion equation, Inverse Problems, 35 (2019), 065004.

[29] T. KÄRKKÄINEN, Error estimates for distributed parameter identification in parabolic problems with output least squares and Crank-Nicolson method, Appl. Math., 42 (1997), pp. 259-277, https://doi.org/10.1023/A:1023012328209.

[30] T. Kato, Fractional powers of dissipative operators, J. Math. Soc. Japan, 13 (1961), pp. 246274, https://doi.org/10.2969/jmsj/01330246.

[31] Y. L. Keung AND J. Zou, Numerical identifications of parameters in parabolic systems, Inverse Problems, 14 (1998), pp. 83-100, https://doi.org/10.1088/0266-5611/14/1/009.

[32] Y. Kian, L. OKSANEn, E. SocCORsi, AND M. YAMAmoto, Global uniqueness in an inverse problem for time fractional diffusion equations, J. Differential Equations, 264 (2018), pp. 11461170, https://doi.org/10.1016/j.jde.2017.09.032.

[33] A. A. Kilbas, H. M. Srivastava, and J. J. Trujillo, Theory and Applications of Fractional Differential Equations, Elsevier Science B.V., Amsterdam, 2006.

[34] S. C. Kou, Stochastic modeling in nanoscale biophysics: Subdiffusion within proteins, Ann. Appl. Stat., 2 (2008), pp. 501-535.

[35] S. C. Kou And X. S. XIE, Generalized Langevin equation with fractional Gaussian noise: Subdiffusion within a single protein molecule, Phys. Rev. Lett., 93 (2004), 180603.

Copyright $@$ by SIAM. Unauthorized reproduction of this article is prohibited. 
[36] G. LI, W. GU, AND X. JiA, Numerical inversions for space-dependent diffusion coefficient in the time fractional diffusion equation, J. Inverse Ill-Posed Probl., 20 (2012), pp. 339-366, https://doi.org/10.1515/jip-2011-0012.

[37] G. Li, D. Zhang, X. Jia, AND M. Yамамото, Simultaneous inversion for the space-dependent diffusion coefficient and the fractional order in the time-fractional diffusion equation, Inverse Problems, 29 (2013), 065014, https://doi.org/10.1088/0266-5611/29/6/065014.

[38] X. Li And C. Xu, A space-time spectral method for the time fractional diffusion equation, SIAM J. Numer. Anal., 47 (2009), pp. 2108-2131, https://doi.org/10.1137/080718942.

[39] Y. Liu, W. Rundell, And M. Yamamoto, Strong maximum principle for fractional diffusion equations and an application to an inverse source problem, Fract. Calc. Appl. Anal., 19 (2016), pp. 888-906, https://doi.org/10.1515/fca-2016-0048.

[40] H. Lou, Optimality conditions for semilinear parabolic equations with controls in leading term, ESAIM Control Optim. Calc. Var., 17 (2011), pp. 975-994, https://doi.org/10.1051/cocv/ 2010034.

[41] R. Metzler, J. H. Jeon, A. G. Cherstvy, and E. Barkai, Anomalous diffusion models and their properties: non-stationarity, non-ergodicity, and ageing at the centenary of single particle tracking, Phys. Chem. Chem. Phys., 16 (2014), pp. 24128-24164.

[42] R. Metzler and J. Klafter, The random walk's guide to anomalous diffusion: A fractional dynamics approach, Phys. Rep., 339 (2000), pp. 1-77.

[43] R. R. Nigmatuluin, The realization of the generalized transfer equation in a medium with fractal geometry, Phys. Stat. Solid. B, 133 (1986), pp. 425-430.

[44] T. K. Nilssen ANd X. C. TAI, Parameter estimation with the augmented Lagrangian method for a parabolic equation, J. Optim. Theory Appl., 124 (2005), pp. 435-453, https://doi. org/10.1007/s10957-004-0944-y.

[45] I. Podlubny, Fractional Differential Equations, Academic Press, San Diego, 1999.

[46] K. Ritchie, X.-Y. Shan, J. Kondo, K. Iwasawa, T. Fujiwara, and A. Kusumi, Detection of non-Brownian diffusion in the cell membrane in single molecule tracking, Biophys. J., 88 (2005), pp. 2266-2277.

[47] K. Sакамото And M. Yамамото, Initial value/boundary value problems for fractional diffusion-wave equations and applications to some inverse problems, J. Math. Anal. Appl., 382 (2011), pp. 426-447, https://doi.org/10.1016/j.jmaa.2011.04.058.

[48] H. Scher And E. W. Montroll, Anomalous transit-time dispersion in amorphous solids, Phys. Rev. B, 12 (1975), pp. 2455-2477.

[49] X.-C. TAI AND T. KÄRKKÄINEN, Identification of a nonlinear parameter in a parabolic equation from a linear equation, Mat. Apl. Comput., 14 (1995), pp. 157-184.

[50] L. WANG AND J. ZOU, Error estimates of finite element methods for parameter identifications in elliptic and parabolic systems, Discrete Contin. Dyn. Syst. Ser. B, 14 (2010), pp. 16411670, https://doi.org/10.3934/dcdsb.2010.14.1641.

[51] T. WEI AND Y. S. LI, Identifying a diffusion coefficient in a time-fractional diffusion equation, Math. Comput. Simulation, 151 (2018), pp. 77-95, https://doi.org/10.1016/j.matcom.2018. 03.006 .

[52] Z. ZHANG, An undetermined coefficient problem for a fractional diffusion equation, Inverse Problems, 32 (2016), 015011, https://doi.org/10.1088/0266-5611/32/1/015011.

[53] Z. Zhang AND Z. ZHOU, Recovering the potential term in a fractional diffusion equation, IMA J. Appl. Math., 82 (2017), pp. 579-600, https://doi.org/10.1093/imamat/hxx004.

Copyright (c) by SIAM. Unauthorized reproduction of this article is prohibited. 